\title{
[18F]Fluorobenzoyllysinepentanedioic Acid Carbamates: New Scaffolds for Positron Emission Tomography (PET) Imaging of Prostate-Specific Membrane Antigen (PSMA)
}

\author{
Xing Yang ${ }^{\dagger} \S$, Ronnie C. Mease ${ }^{\dagger, \S}$, Mrudula Pullambhatla $^{\dagger}$, Ala Lisok $^{\dagger}$, Ying Chen $^{\dagger}$, \\ Catherine A. Foss ${ }^{\dagger}$, Yuchuan Wang ${ }^{\dagger}$, Hassan Shallal ${ }^{\dagger}$, Hannah Edelman ${ }^{\dagger}$, Adam T. Hoye ${ }^{\ddagger}$, \\ Giorgio Attardo ${ }^{\ddagger}$, Sridhar Nimmagadda ${ }^{\dagger}$, and Martin G. Pomper ${ }^{\star}, \dagger$ \\ †Russell H. Morgan Department of Radiology and Radiological Science, Johns Hopkins Medical \\ Institutions, Baltimore, Maryland 21287, United States \\ ${ }^{\ddagger}$ Avid Radiopharmaceuticals, Inc., Philadelphia, Pennsylvania 19104, United States
}

\begin{abstract}
Radiolabeled urea-based low-molecular weight inhibitors of the prostate-specific membrane antigen (PSMA) are under intense investigation as imaging and therapeutic agents for prostate and other cancers. In an effort to provide agents with less nontarget organ uptake than the ureas, we synthesized four ${ }^{18} \mathrm{~F}$-labeled inhibitors of PSMA based on carbamate scaffolds. 4-Bromo-2$\left[{ }^{18} \mathrm{~F}\right]$ fluorobenzoyllysineoxypentanedioic acid (OPA) carbamate $\left[{ }^{18} \mathrm{~F}\right] 23$ and 4-iodo-2$\left[{ }^{18} \mathrm{~F}\right]$ fluorobenzoyllysine OPA carbamate $\left[{ }^{18} \mathrm{~F}\right] 24$ in particular exhibited high target-selective uptake in PSMA+ PC3 PIP tumor xenografts, with tumor-to-kidney ratios of $>1$ by $4 \mathrm{~h}$ postinjection, an important benchmark. Because of its high tumor uptake (90\% injected dose per gram of tissue at $2 \mathrm{~h}$ postinjection) and high tumor-to-organ ratios, $\left[{ }^{18} \mathrm{~F}\right] 23$ is promising for clinical translation. Prolonged tumor-specific uptake demonstrated by $\left[{ }^{18} \mathrm{~F}\right] 24$, which did not reach equilibrium during the $4 \mathrm{~h}$ study period, suggests carbamates as alternative scaffolds for mitigating dose to nontarget tissues.
\end{abstract}

\section{Graphical Abstract}

*Corresponding Author: Phone: 410-955-2789. Fax: 443-819-0990. mpomper@jhmi.edu.

$\$$ X.Y. and R.C.M. contributed equally.

\section{ASSOCIATED CONTENT}

Supporting Information

The Supporting Information is available free of charge on the ACS Publications website at DOI: 10.1021/acs.jmedchem.5b01268.

Additional chemical syntheses, ${ }^{1} \mathrm{H}$ NMR, mass spectrometry, radiochemical methods, and radio-HPLC (PDF)

The authors declare no competing financial interest. 


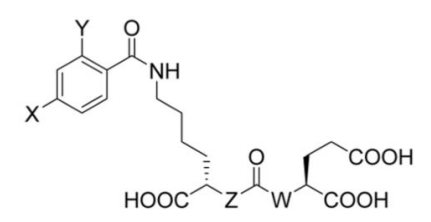

9 $\mathrm{X}=\mathrm{F}, \mathrm{Y}=\mathrm{H}, Z=\mathrm{NH}, W=\mathrm{NH} \quad \mathrm{K}_{\mathrm{i}}=0.25 \mathrm{nM}$ $12 X=F, Y=H, Z=O, W=N H \quad K_{i}=42 n M$ $23 X=B r, Y=F, Z=N H, W=O \quad K_{i}=0.11 n M$

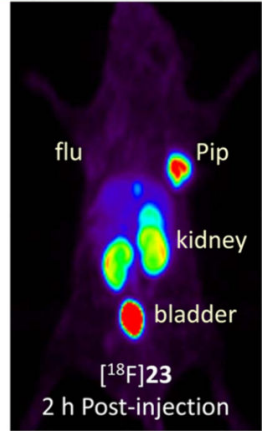

\section{INTRODUCTION}

Prostate-specific membrane antigen (PSMA) is among the most intensively targeted biomarkers for imaging metastatic prostate cancer. PSMA is a zinc-dependent metallopeptidase that catalyzes the hydrolysis of a series of $N$-acylpoly- $\gamma$-glutamate derivatives. ${ }^{1}$ It is expressed within certain normal tissues ${ }^{2}$ but transitions to abundant plasma membrane expression in the epithelium of most prostate cancers and within other solid tumor neovasculature. ${ }^{3}$ PSMA membrane expression is associated with metastasis, ${ }^{4}$ castration resistance, ${ }^{5}$ and progression of prostate cancer. ${ }^{6}$

Several different scaffolds are available for synthesis of small-molecule PSMA inhibitors. Those have been reviewed. ${ }^{7}$ As shown in Figure 1, those potent scaffolds share common features, namely, (a) a pentanedioic acid (green) as a glutamate mimic to fit within the $\mathrm{S} 1^{\prime}$ binding pocket of the active site; (b) a zinc-binding group (blue) to interact with the catalytic zinc atom at the PSMA active site. A substituent $(\mathrm{R})$ can reside either within the S1 binding pocket or within a void in the protein that extends to the surface. Scaffolds composed of phosphonates or phosphinates, ${ }^{8}$ phosphoramidates, ${ }^{9}$ and ureas ${ }^{9,10}$ of general structures 1-3, as well as thiol $\mathbf{4}^{11}$ and hydroxamate $\mathbf{5 ,}{ }^{12}$ have been reported as effective zinc binding groups for PSMA inhibition. However, the presence of a zinc binding moiety and a glutamate mimic residing in the $\mathrm{S1}^{\prime}$ pocket is not sufficient for high binding as demonstrated by Gly-urea-Glu compound $\mathbf{8}$ (Figure 2; Table 1). ${ }^{10 a}$ Of the reported PSMA binding scaffolds, urea-based inhibitors, first introduced by Kozikowski in 2001 $10 \mathrm{a}$ for inhibition of glutamate carboxypeptidase II within the central nervous system, have been utilized the most for targeting PSMA due to their high binding affinity and synthetic simplicity. ${ }^{10 a}{ }^{13} \mathrm{~A}$ variety of low-molecular-weight compounds based on the various scaffolds discussed above, primarily the ureas, have been labeled with radionuclides for positron emission tomography (PET) and single photon emission computed tomography (SPECT), namely, ${ }^{125 / 124} \mathrm{I},{ }^{99 \mathrm{~m}} \mathrm{Tc},{ }^{111} \mathrm{In},{ }^{18} \mathrm{~F},{ }^{11} \mathrm{C},{ }^{68} \mathrm{Ga},{ }^{64} \mathrm{Cu}$, and ${ }^{86} \mathrm{Y}$, and have demonstrated PSMAtargeted imaging of prostate cancer in experimental models. ${ }^{13 \mathrm{a}-\mathrm{d}, 14}$ Several of those have been translated to phase $0-1$ clinical trials, where they have enabled visualization of both primary and metastatic bone and soft-tissue lesions due to prostate cancer. ${ }^{15}$

However, clinical imaging studies also exhibited considerable uptake in nontarget PSMAexpressing tissues such as the salivary glands and kidneys, revealing potential dose-limiting off-target effects, particularly for radiotherapeutic analogs. We sought additional PSMA- 
binding scaffolds that might preserve the positive imaging characteristics of the ureido scaffolds but clear from the nontarget organs. We chose the carbamate scaffold because it would retain the overall geometry of the existing inhibitors, differing only with an $\mathrm{O}$ for $\mathrm{NH}$ substitution, which eliminates a potential hydrogen bonding group present in the ureas. The only PSMA-binding carbamate reported is Gly-aminopentanedioic acid 11. ${ }^{16}$ It displayed low binding affinity to PSMA, most likely due to the absence of productive binding within the $\mathrm{S} 1$ pocket, similar to ureido compound $\mathbf{8}$.

Here we report a new class of potent PSMA inhibitors based on the carbamate scaffold to maintain glutamate and S1 pocket side chain geometry and for potential binding to zinc. Carbamates of general structure $\mathbf{6}$ contain the amino-pentanedioic acid (NPA) moiety, whereas those of general structure 7 contain the oxypentanedioic acid moiety (OPA) (Figure $1)$.

\section{RESULTS}

\section{Chemistry}

Among the potent urea-based PSMA-binding ligands, 4-fluorobenzoyl- and 4-iodobenzoylLys-Glu urea, compounds $\mathbf{9}$ and 10, respectively (Figure 2), have produced some of the highest affinities reported (Table 1). ${ }^{13 \mathrm{~d}}$ On the basis of those ureas, NPA- and OPAcarbamates 12 and $\mathbf{1 3}$ (Schemes 1 and 2) were our initial target compounds. We reasoned that the 4-fluorobenzoyl side chain would provide high affinity and specificity by utilizing the $\mathrm{S} 1$ binding pocket and be amenable to radiolabeling with ${ }^{18} \mathrm{~F}$. The synthesis of $\mathbf{1 2}$ started with $N$-Boc protected (S)-6-amino-2-hydroxyhexanoic acid 14 (Scheme 1). We first protected the carboxylic acid as the tert-butyl ester and then converted alcohol 15 to $\mathrm{N}$ imidazolecarbamate 16. Compound 16 reacted slowly with L-glutamic acid di-tert-butyl ester under neat conditions and provided a quantitative yield of $\mathbf{1 7}$. Upon deprotection intermediate $\mathbf{1 8}$ was obtained in 70\% yield as the trifluoroacetate salt. NPA-carbamate $\mathbf{1 2}$ was obtained by treating 18 with $N$-succinimidyl-4-fluorobenzoate in the presence of triethylamine.

OPA carbamate $\mathbf{1 3}$ was prepared from $(S)$-dimethyl-2-hydroxypentanedioate 19, for which the corresponding tert-butyl ester was not easily accessible (Scheme 2). After conversion of 19 to the $N$-imidazolecarbamate, 20 was first transformed to its imidazolium salt via treatment with iodomethane and then reacted with $N^{\varepsilon}$-Boc-lysine-tert-butyl ester to afford 21. Attempts to couple $N^{E}$-boc-lysine-tert-butyl ester and $\mathbf{2 0}$ directly failed to give the desired product, presumably due to side reactions involving the methyl esters. After two deprotection steps amine $\mathbf{2 2}$ was conjugated with $\mathrm{N}$-succinimidyl-4-fluorobenzoate to give OPA-carbamate 13.

X-ray cocrystal studies with bound urea-based ligands such as $\mathbf{1 0}$ have demonstrated a unique cation- $\pi$ interaction with PSMA, with the benzoyl group of the ligand fully inserted into the "arginine patch" of the PSMA S1 binding pocket and an adjacent hydrophobic subpocket that can accommodate the para-iodo substituent of 10. ${ }^{13 \mathrm{e}}$ That additional interaction likely accounted for the increase in binding affinity of $\mathbf{1 0}$ compared to $\mathbf{9} .{ }^{13 \mathrm{de}}$ To increase the binding affinities of the carbamates, we likewise prepared iodinated carbamates 
31 and 32 by reacting $N$-succinimidyl-4-iodobenzoate with 18 and 22, respectively (Schemes 1 and 2). For PET imaging with ${ }^{18} \mathrm{~F}$, the 4-iodobenzoyl group would require an $\left[{ }^{18}\right.$ F $]$ fluoro substituent in the activated ortho position. With that in mind we generated OPA carbamates $\mathbf{2 3}$ and $\mathbf{2 4}$ by reacting $\mathbf{2 2}$ with $\mathrm{N}$-succinimidyl-4-bromo/iodo-2-fluorobenzoate (Scheme 2). For comparison, we also synthesized the corresponding ureido analogs $\mathbf{2 6}$ and 27 from urea 25 (Scheme 2). ${ }^{14 k, 17}$

\section{In Vitro PSMA Binding}

The PSMA inhibitory activities of the prepared compounds were measured using a modification of the fluorescence-based Amplex red glutamic acid assay ${ }^{10 \mathrm{~b}}$ (Table 1). Carbamates 12 and 13 inhibited PSMA at $K_{\mathrm{i}}=42 \mathrm{nM}$ and $K_{\mathrm{i}}=9.2 \mathrm{nM}$, respectively, significantly less potent than the corresponding ureido analog $9\left(K_{\mathrm{i}}=0.25 \mathrm{nM}\right) .{ }^{13 \mathrm{~d}}$ Carbamates 31 and 32 inhibited PSMA at $K_{\mathrm{i}}=0.9 \mathrm{nM}$ and $K_{\mathrm{i}}=0.04 \mathrm{nM}$, respectively. Carbamates 23 and 24 demonstrated potent inhibition of PSMA, with $K_{\mathrm{i}}=0.11 \mathrm{nM}$ and $K_{\mathrm{i}}=$ $0.21 \mathrm{nM}$, respectively, while the corresponding ureas 26 and 27 inhibited PSMA with $K_{\mathrm{i}}=$ 0.04 and $K_{\mathrm{i}}=0.02 \mathrm{nM}$, respectively.

\section{PSMA Inhibitor Docking Studies}

Urea 9, NPA-carbamate 12, and OPA-carbamate 13 were subjected to in situ ligand minimization docking experiments based on the reported X-ray crystal structure of PSMA, known as PDB structure 3D7H. ${ }^{13 \mathrm{e}}$ The results are shown in Figure 3. The urea N-H in 9 binds to the carbonyl oxygen of G518 asymmetrically, with the Lys-N-H (1.92 A) slightly closer to the carbonyl oxygen than Glu-N-H (2.03 ̊) (Figure 3A). Lys-OPA carbamate 13, with the Glu-N-H changed to $\mathrm{O}$ resulting in the loss of a hydrogen bonding group, retained nearly all of the features of 9 when binding to PSMA (Figure 3C). The Lys-N-H distance to G518 slightly increased to $1.95 \AA$ with the carbamate shifting slightly toward the zinc (Figure $3 \mathrm{C}$ vs Figure $3 \mathrm{~A}$ ). That resulted in a 30 -fold reduction in binding affinity compared to 9. NPA carbamate 12, with the Lys-N-H changed to $\mathrm{O}$, altered the binding mode dramatically (Figure 3B) with the carbamate shifting toward the zinc resulting in an increase in hydrogen bond distance to G518 (2.448 $\AA$ ) (Figure 3B). The binding affinity of $\mathbf{1 2}$ was decreased by more than 150 -fold compared to 9 .

\section{Radiochemistry}

Carbamates $\left[{ }^{18} \mathrm{~F}\right] \mathbf{1 2}$ and $\left[{ }^{18} \mathrm{~F}\right] \mathbf{1 3}$ were prepared by reacting $N$-succinimidyl-4$\left[{ }^{18} \mathrm{~F}\right]$ fluorobenzoate, $\left[{ }^{18} \mathrm{~F}\right] \mathrm{SFB},{ }^{18}$ with $\mathbf{1 8}$ and $\mathbf{2 2}$, respectively. ${ }^{125}$ I-Labeled analogs $\mathbf{3 1}$ and 32 (Schemes 1 and 2) were prepared likewise using $N$-succinimidyl-4-[ ${ }^{125}$ I]iodobenzoate. ${ }^{19}$ To synthesize $\left[{ }^{18} \mathrm{~F}\right] \mathbf{2 3}$ and $\left[{ }^{18} \mathrm{~F}\right] \mathbf{2 4}$, we envisioned the conjugation of $N$-succinimidyl-4bromo/iodo-2- $\left[{ }^{18} \mathrm{~F}\right]$ fluorobenzoate $\left(\left[{ }^{18} \mathrm{~F}\right] \mathbf{3 0 b}\right.$ and $\left.\left[{ }^{18} \mathrm{~F}\right] \mathbf{3 0 c}\right)$ with precursor 22 (Scheme 3). Compared with the synthesis of $4-\left[{ }^{18} \mathrm{~F}\right]$ fluorobenzoate and $2-\left[{ }^{18} \mathrm{~F}\right]$ fluorobenzoate derivatives, the synthesis of 4-bromo/iodo-2-[ $\left.{ }^{18} \mathrm{~F}\right]$ fluorobenzoate derivatives has inherent challenges. The bromo/iodo moiety could potentially serve as a leaving group and be displaced by $\left[{ }^{18} \mathrm{~F}\right]$ fluoride, producing a radiofluorinated side product. The only related example reported previously was the synthesis of 4-chloro-2-[ $\left.{ }^{18} \mathrm{~F}\right]$ fluorobenzaldehyde 29a from 4-chloro-2-nitro-benzaldehyde $\mathbf{2 8 a}$ (Scheme 3), which proceeded in a $60 \%$ 
radiochemical yield using Kryptofix 2.2 .2 and $\mathrm{K}_{2} \mathrm{CO}_{3}$ in DMSO at $140{ }^{\circ} \mathrm{C}$ for 20 min. ${ }^{20}$ As the first step in the synthesis of $\left[{ }^{18} \mathrm{~F}\right] \mathbf{2 3}$ and $\left[{ }^{18} \mathrm{~F}\right] \mathbf{2 4}$, we applied those reaction conditions to the radiosynthesis of 4-bromo- and 4-iodo-2-[ $\left.{ }^{18} \mathrm{~F}\right]$-fluorobenzaldehyde $29 \mathbf{b}$ and $29 \mathrm{c}$, starting from 4-bromo- and 4-iodo-2-nitrobenzaldehyde, $\mathbf{2 8 b}$ and $\mathbf{2 8 c}$, respectively (Scheme 3). Compared with the reported success of $\left[{ }^{18} \mathrm{~F}\right] 29 \mathrm{a}$, the highest yield that could be achieved for $\left[{ }^{18} \mathrm{~F}\right] \mathbf{2 9 b}$ was only $15 \%$, nondecay corrected yield (Supporting Information Table S1, entries 1-8). No $\left[{ }^{18} \mathrm{~F}\right] \mathbf{2 9 c}$ was produced under those conditions. Under those conditions decomposition of precursors $\mathbf{2 8 b}$ and $\mathbf{2 8 c}$ was observed by high performance liquid chromatography (HPLC). Decomposition was reduced using a milder base, $\mathrm{KHCO}_{3}$, enabling the radiosynthesis of aldehyde $\left[{ }^{18} \mathbf{F}\right] \mathbf{2 9 b}$ and $\left[{ }^{18} \mathbf{F}\right] \mathbf{2 9 c}$. The complete radiosynthesis of $\left[{ }^{18} \mathrm{~F}\right] \mathbf{2 3}$ and $\left[{ }^{18} \mathrm{~F}\right] \mathbf{2 4}$ is shown in Scheme 3 . Compound $\left[{ }^{18} \mathrm{~F}\right] \mathbf{2 9 b}$ was converted to its $N$ hydroxysuccinimide ester $\left[{ }^{18} \mathrm{~F}\right] \mathbf{3 0 b}$ by treatment with (diacetoxyiodo)benzene in the presence of $N$-hydroxysuccinimide in acetonitrile. ${ }^{21}$ After conjugating the ${ }^{18} \mathrm{~F}$-labeled NHS ester with Lys-OPA carbamate precursor $\mathbf{2 2},\left[{ }^{18} \mathrm{~F}\right] \mathbf{2 3}$ was obtained in a nondecay corrected radiochemical yield of $5 \%$ and specific radioactivities ranging from 37 to $1776 \mathrm{GBq} / \mu \mathrm{mol}$ $(1000-4800 \mathrm{Ci} / \mathrm{mmol})$. Compound $\left[{ }^{18} \mathrm{~F}\right] \mathbf{2 4}$ was prepared in the same fashion $(3 \%$ nondecay corrected yield; specific radioactivities ranging between 31.1-925 GBq/ $\mu \mathrm{mol}(840-2500 \mathrm{Ci} /$ mmol).

\section{Biodistribution and Imaging}

Compounds $\left[{ }^{18} \mathrm{~F}\right] \mathbf{1 2},\left[{ }^{18} \mathrm{~F}\right] \mathbf{1 3},\left[{ }^{18} \mathrm{~F}\right] \mathbf{2 3}$, and $\left[{ }^{18} \mathrm{~F}\right] \mathbf{2 4}$ were assessed for their tumor uptake and pharmacokinetics in mice bearing PSMA+ PC3 PIP and PSMA- PC3 flu xenografts by ex vivo biodistribution and/or small animal PET imaging. Whole body small animal PET/CT images of $\left[{ }^{18} \mathrm{~F}\right] \mathbf{1 2}$ are shown in Figure 4, with the corresponding biodistribution shown in Table 2. Compound $\left[{ }^{18} \mathrm{~F}\right] \mathbf{1 2}$ exhibited a modest PSMA+ PC3 PIP tumor uptake of 6.33 \pm 1.55 percentage injected dose per gram of tissue $(\% \mathrm{ID} / \mathrm{g})$ at $30 \mathrm{~min}$, and radioactivity rapidly cleared from all organs including tumor. PET/CT imaging confirmed the rapid tumor uptake and clearance from all tissues. Co-injection of $\left[{ }^{18} \mathrm{~F}\right] \mathbf{1 2}$ with an excess of known competing inhibitor (S)-2-(3-((S)-1- carboxy-3-methylbutyl)ureido)pentanedioic acid $(\mathrm{ZJ}-43)^{22}$ showed complete blockade of tumor uptake indicating that the uptake was PSMAmediated. Small animal PET imaging of $\left[{ }^{18} \mathrm{~F}\right] \mathbf{1 3}$ (Figure 5) showed higher and more prolonged PSMA+ PC3 PIP tumor and kidney uptake than $\left[{ }^{18} \mathrm{~F}\right] \mathbf{1 2}$, reminiscent of ureido compound $\left[{ }^{18} \mathrm{~F}\right] 9$, previously reported by us. ${ }^{13 \mathrm{~d}}$

Table 3 shows the $\%$ ID/g in selected organs for $\left[{ }^{18} \mathrm{~F}\right] 23$. Compound $\left[{ }^{18} \mathrm{~F}\right] \mathbf{2 3}$ demonstrated high uptake in the PSMA+ PC3 PIP tumor, wherein the uptake of radioactivity reached a maximum of $90 \% \mathrm{ID} / \mathrm{g}$ at $2 \mathrm{~h}$ postinjection. On the contrary, the PSMA- PC3 flu tumor showed no specific uptake. The distribution within nontarget tissues was generally low except for kidney, liver and spleen. Significant renal uptake was observed, which can be attributed to the expression of PSMA in the proximal renal tubules with concurrent renal excretion. ${ }^{23}$ Radioactivity cleared from the kidneys and by $4 \mathrm{~h}$ was less than the level in PSMA+ PC3 PIP tumor. Other than kidney, the highest nonspecific uptake was observed in liver and spleen at $30 \mathrm{~min}$, both of which demonstrated rapid clearance of radiotracer, reaching minimal levels by $4 \mathrm{~h}$. Low radiotracer uptake within bone indicated lack of significant defluorination in vivo. The PSMA+ PC3 PIP:muscle and PSMA+ PC3 PIP:blood 
ratios were 93 and 13 at $0.5 \mathrm{~h}, 208$ and 40 at $1 \mathrm{~h}, 643$ and 148 at $2 \mathrm{~h}$, and 792 and 240 at $4 \mathrm{~h}$, respectively.

The biodistribution of $\left[{ }^{18} \mathrm{~F}\right] \mathbf{2 4}$ is shown in Table 4. Blood, other organs, and normal tissues displayed relatively low uptake and rapid clearance. Kidneys showed high initial uptake with clearance beginning after $2 \mathrm{~h}$ and was less than the uptake in PSMA+ PC3 PIP tumor by $4 \mathrm{~h}$. The PSMA+ PC3 PIP:muscle and PSMA+ PC3 PIP:blood ratios were 47 and 5.6 at $0.5 \mathrm{~h}$, 100 and 20 at 1 h, 250 and 66 at 2h, and 743 and 156 at 4 h, respectively.

Whole body small animal PET imaging was also performed for $\left[{ }^{18} \mathrm{~F}\right] \mathbf{2 3}$ and $\left[{ }^{18} \mathrm{~F}\right] \mathbf{2 4}$. Intense and selective radiotracer uptake could be seen in PSMA+ PC3 PIP tumors beginning at 30 min postinjection (Figure 6). Concordant with the biodistribution results, kidneys also had significant uptake. Besides PSMA+ PC3 PIP tumor, kidneys, and bladder, no significant nonspecific organ uptake was observed.

\section{In Vitro Stability}

Because NPA and OPA carbamates $\left[{ }^{18} \mathrm{~F}\right] \mathbf{1 2}$ and $\left[{ }^{18} \mathrm{~F}\right] \mathbf{1 3}$ exhibited such diverse pharmacokinetics, we tested the stability of NPA and OPA carbamates $\left[{ }^{125} \mathrm{I}\right] \mathbf{3 1}$ and $\left[{ }^{125} \mathrm{I}\right] \mathbf{3 2}$ in vitro. The radioiodinated compounds were utilized for the convenience of the long physical half-life of ${ }^{125} \mathrm{I}$.

Each agent was incubated with PSMA+ PC3 PIP and PSMA- PC3 flu cells. Extracellular fluid medium and intracellular extracts were analyzed by radioactive thin layer chromatography. Polar radiometabolites in extracellular and intracellular fluid were only seen for NPA carbamate 33 in PSMA+ PC3 PIP cells (Figure S1, Supporting Information). The data suggest that NPA carbamates may be subject to PSMA-specific metabolism, which may account for their rapid clearance from PSMA expressing tumors and organs.

\section{DISCUSSION}

Recent clinical PET imaging using ${ }^{18} \mathrm{~F}-,{ }^{124} \mathrm{I}$-, or ${ }^{68} \mathrm{Ga}$-labeled, urea-based PSMA inhibitors has produced high quality PET images allowing the detection of both primary and metastatic lesions, some of which were undetectable by conventional imaging. Although all agents exhibited renal uptake, some agents, e.g., $\left[{ }^{18}\right.$ F $]$ DCFBC ${ }^{15 f}$ and $\left[{ }^{68}\right.$ Ga]DOTA-DUPA-Pep, ${ }^{24}$ showed persistent blood pool occupancy but low salivary gland uptake. On the other hand, $\left[{ }^{18} \mathrm{~F}\right] \mathrm{DCFPyL},{ }^{15 \mathrm{~h}}\left[{ }^{124} \mathrm{I}\right] \mathrm{MIP}-1095,{ }^{25}$ and $\left[{ }^{68} \mathrm{Ga}\right] \mathrm{DKFZ}-11{ }^{15 b, 26}$ showed rapid clearance from the blood but high uptake in salivary and lacrimal glands. The latter has also been observed in therapeutic studies using $\left[{ }^{177} \mathrm{Lu}\right] \mathrm{DKFZ}-617^{27}$ and $\left[{ }^{131} \mathrm{I}\right] \mathrm{MIP}-1095 .{ }^{25}$ Absorbed dose estimates for $\left[{ }^{131}\right.$ I]MIP-1095 identified the salivary glands, lower large intestinal wall, and kidneys as dose-limiting organs. ${ }^{25}$ We sought new scaffolds that would preserve the positive distribution characteristics of the urea-based agents but clear from nontarget tissues. The carbamate-based agents described here remained potent and also rapidly cleared from normal tissues. However, NPA carbamate $\left[{ }^{18} \mathrm{~F}\right] \mathbf{1 2}$ and OPA carbamate $\left[{ }^{18} \mathrm{~F}\right] \mathbf{1 3}$ exhibited significantly different pharmacokinetics: $\left[{ }^{18} \mathrm{~F}\right] \mathbf{1 2}$ had modest PSMA+ PC3 PIP tumor uptake but rapidly cleared from both PSMA+ PC3 PIP tumor and normal tissues including kidneys, whereas OPA carbamate $\left[{ }^{18} \mathrm{~F}\right] 13$ had high and prolonged tumor and kidney uptake. 
Selective metabolism of NPA carbamate $\left[{ }^{125} \mathrm{I}\right] 31$ was observed only in PSMA+ PC3 PIP cells in vitro, suggesting that NPA carbamates may be PSMA substrates. In situ ligand minimization docking experiments showed a difference in the binding modes of $\mathbf{1 2}$ and $\mathbf{1 3}$. Although 12 and 13 each have one less hydrogen bonding group compared to urea 9, 13 possesses a similar position with respect to zinc and G518 in the PSMA binding site. For 12 the hydrogen bonding distance to G518 is increased. The resulting weaker hydrogen bonding in $\mathbf{1 2}$ may account for its lower PSMA binding affinity compared to both $\mathbf{9}$ and $\mathbf{1 3}$.

Higher potency Lys-OPA carbamates $23\left(K_{\mathrm{i}}=0.11 \mathrm{nM}\right)$ and $\mathbf{2 4}\left(K_{\mathrm{i}}=0.21 \mathrm{nM}\right)$ were obtained by utilization of 4-bromo/ iodo-2-fluorobenzoyl groups, which increased PSMA binding presumably through interaction with the arginine patch and the auxiliary hydrophobic subpocket. ${ }^{13 e}$ Compounds $\mathbf{2 3}$ and $\mathbf{2 4}$ bound with less than a 10-fold decrease in potency compared with the corresponding ureas $26\left(K_{\mathrm{i}}=0.036 \mathrm{nM}\right)$ and $27\left(K_{\mathrm{i}}=0.023\right.$ $\mathrm{nM}$ ). That suggests that utilization of the $\mathrm{S} 1$ binding site (arginine patch) and the auxiliary hydrophobic pocket can partially offset the loss of one hydrogen bond in the OPA carbamates compared to ureas.

The biodistribution and imaging data demonstrated specific binding of Lys-OPA carbamates $\left[{ }^{18} \mathrm{~F}\right] \mathbf{2 3}$ and $\left[{ }^{18} \mathrm{~F}\right] \mathbf{2 4}$ to PSMA. Little bone uptake suggested the stability of the $\left[{ }^{18} \mathrm{~F}\right]$ fluorine on the 4-bromo/iodo-2- $\left[{ }^{18} \mathrm{~F}\right]$ fluorobenzoyl group. Although there was relatively little uptake of $\left[{ }^{18} \mathrm{~F}\right] \mathbf{2 3}$ and $\left[{ }^{18} \mathrm{~F}\right] \mathbf{2 4}$ within blood pool, it was somewhat higher than for similar compounds previously published, such as $\left[{ }^{125} \mathrm{I}\right] \mathbf{1 0}{ }^{13 \mathrm{~d}}$ and $\left[{ }^{18} \mathrm{~F}\right] \mathrm{DCFPyL},{ }^{15 \mathrm{~h}}$ possibly due to the greater lipophilicity imparted by the large halogens on the aromatic ring in addition to fluorine. For $\left[{ }^{18} \mathrm{~F}\right] \mathbf{2 3}$ the initial PSMA+ PC3 PIP tumor uptake at $30 \mathrm{~min}$ was $63 \% \mathrm{ID} / \mathrm{g}$, increasing to $90 \% \mathrm{ID} / \mathrm{g}$ at $2 \mathrm{~h}$ and then dropped slightly to $74 \% \mathrm{ID} / \mathrm{g}$ at $4 \mathrm{~h}$. In the case of $\left[{ }^{18} \mathrm{~F}\right] 24$, the initial PSMA+ PC3 PIP tumor uptake observed was $45 \% \mathrm{ID} / \mathrm{g}$, increasing to $97 \% \mathrm{ID} / \mathrm{g}$ by $4 \mathrm{~h}$. Both radiotracers showed little uptake in the isogenic, PSMA- PC3 flu tumors. For blood and normal organs the highest nonspecific uptake appeared in liver and spleen, but this radioactivity cleared rapidly resulting in increasing tumor/normal organ ratios. Although $\left[{ }^{18} \mathrm{~F}\right] \mathbf{2 3}$ and $\left[{ }^{18} \mathrm{~F}\right] \mathbf{2 4}$ still possess considerable renal uptake, their tumor/ kidney ratios are approximately 1 at $2-4 \mathrm{~h}$ and are $4-10$ times higher than the ratios we previously reported for ureas $\left[{ }^{18} \mathrm{~F}\right] \mathbf{9}$ and $\left[{ }^{125} \mathrm{I}\right] \mathbf{1 0} .{ }^{13 \mathrm{~d}}$ Interestingly, $\left[{ }^{18} \mathrm{~F}\right] \mathbf{2 3}$ and $\left[{ }^{18} \mathrm{~F}\right] \mathbf{2 4}$ are more potent than our second generation ${ }^{18} \mathrm{~F}$-labeled PSMA inhibitor, $\left[{ }^{18} \mathrm{~F}\right] \mathrm{DCFPyL}\left(K_{\mathrm{i}}=\right.$ $1.1 \mathrm{nM}$ ), currently in clinical trials. ${ }^{15 \mathrm{~h}}$ However, in PSMA+ PC3 PIP tumor-bearing mice $\left[{ }^{18} \mathrm{~F}\right]$-DCFPyL demonstrated higher tumor:kidney ratios ( $>2$ at $2 \mathrm{~h}$ postinjection). ${ }^{14 \mathrm{e}}$ Neither $\left[{ }^{18} \mathrm{~F}\right] \mathbf{2 3}$ nor $\left[{ }^{18} \mathrm{~F}\right] \mathbf{2 4}$ demonstrated any uptake in salivary glands on imaging. Clinical imaging studies will be necessary to determine if OPA carbamates offer any improvement over $\left[{ }^{18}\right.$ F $]$ DCFPyL, particularly since we did not see salivary uptake for $\left[{ }^{18} \mathrm{~F}\right] \mathrm{DCFPyL}$ in the preclinical model either. The OPA scaffold may be most useful for radiotherapy with $a, \beta$, or Auger electron emitters when labeled with radiohalogenated ${ }^{211} \mathrm{At},{ }^{131} \mathrm{I},{ }^{125} \mathrm{I},{ }^{123} \mathrm{I},{ }^{80 \mathrm{~m}} \mathrm{Br}$, or ${ }^{77} \mathrm{Br}$ ) benzoyl groups, where the $\mathrm{S} 1$ and auxiliary hydrophobic pockets are fully utilized.

The $N$-succinimidyl 4-bromo/iodo-2- $\left[{ }^{18} \mathrm{~F}\right]$ fluorobenzoates $\left[{ }^{18} \mathrm{~F}\right] \mathbf{3 0 b}$ and $\left[{ }^{18} \mathrm{~F}\right] \mathbf{3 0 c}$ utilized in the synthesis of $\left[{ }^{18} \mathrm{~F}\right] \mathbf{2 3}$ and $\left[{ }^{18} \mathrm{~F}\right] \mathbf{2 4}$ are new radiofluorinated prosthetic groups. We recognize that the radiosyntheses of $\left[{ }^{18} \mathrm{~F}\right] \mathbf{2 3}$ and $\left[{ }^{18} \mathrm{~F}\right] \mathbf{2 4}$ reported here provide low overall radiochemical yields and are cumbersome to perform. However, our initial goal was only to 
produce enough radiolabeled material for preliminary testing in vivo. Improved radiochemical yields will be required for $\left[{ }^{18} \mathrm{~F}\right] \mathbf{2 3}$ and $\left[{ }^{18} \mathrm{~F}\right] \mathbf{2 4}$ to achieve their full potential as imaging agents, with such studies underway.

\section{CONCLUSION}

We describe the carbamate scaffold for preparation of potent inhibitors of PSMA. Lys-OPA carbamates were more potent than Lys-NPA carbamates. By utilizing the affinity-enhancing interactions of the arginine patch and auxiliary hydrophobic subpocket, $\left[{ }^{18} \mathrm{~F}\right] \mathbf{2 3}$ and $\left[{ }^{18} \mathrm{~F}\right] \mathbf{2 4}$ demonstrated high, target-selective uptake in PSMA+ PC3 PIP tumor xenografts. Those agents produced higher PSMA+ PC-3 PIP:kidney ratios compared to similar ureas, such as $\left[{ }^{125} \mathrm{I}\right] \mathbf{1 0}$ and $\left[{ }^{18} \mathrm{~F}\right] \mathbf{9}$, suggesting this alternative scaffold for development of new agents with improved dosimetry, particularly for radiotherapeutics. Because of its rapid normal organ clearance, $\left[{ }^{18} \mathrm{~F}\right] \mathbf{2 3}$ is particularly suitable for clinical translation.

\section{EXPERIMENTAL SECTION}

\section{General Procedures}

Solvents and chemicals purchased from commercial sources were of analytical grade or better and used without further purification. tert-Butyl-1,3-diisopropylisourea-L-glutamic acid di-tert-butyl ester, $N^{E}$-Boc-lysine-tert-butyl ester hydrochloride, 1-hydroxybenzotriazole monohydrate, and 2-(1H-benzotriazole-1-yl)-1,1,3,3-tetramethyluronium hexafluorophosphate (HBTU) were purchased from Chem Impex International Inc. (Wooddale, IL). 1,1'-Carbonyldiimidazole, iodomethane, $N$-hydroxysuccinimide, (diacetoxyiodo)benzene, triethylsilane ( $\mathrm{Et}_{3} \mathrm{SiH}$ ), diisopropylethylamine (DIEA), and triethylamine (TEA) were purchased from Sigma-Aldrich (St. Louis, MO). 4-Bromo-2nitrobenzaldehyde was purchased from Combi-Blocks (San Diego, CA). (S)-6-((tertButoxycarbonyl)amino)-2-hydroxyhexanoic acid, ${ }^{28}$ 4-iodo-2-nitroben-zaldehyde, ${ }^{29}$ and $(S)$ dimethyl 2-hydroxypentanedioate ${ }^{30}$ were synthesized by previously reported procedures. Analytical thin-layer chromatography (TLC) was performed using Aldrich aluminumbacked $0.2 \mathrm{~mm}$ silica gel Z19, 329-1 plates and visualized by ultraviolet light ( $254 \mathrm{~nm}), \mathrm{I}_{2}$ and $1 \%$ ninhydrin in EtOH. Flash chromatography was performed using silica gel (MP SiliTech 32-63 D 60 Å) purchased from Bodman (Aston, PA). All in vitro PSMA binding studies were performed in triplicate. ${ }^{1} \mathrm{H}$ NMR spectra were recorded on a Bruker Ultrashield 400 or $500 \mathrm{MHz}$ spectrometer. Chemical shifts $(\delta)$ are reported in ppm downfield by reference to proton resonances resulting from incomplete deuteration of the NMR solvent. After purification all compounds and stable intermediates were proved to be $>95 \%$ in purity by quantitative ${ }^{1} \mathrm{H}$ NMR (Supporting Infomation). Low resolution ESI mass spectra were obtained on a Bruker Daltonics Esquire 3000 Plus spectrometer. High resolution mass spectra were obtained at the University of Notre Dame Mass Spectrometry \& Proteomics Facility, Notre Dame, IN, using ESI either by direct infusion on a Bruker microTOF-II or by LC elution via an ultrahigh pressure Dionex RSLC with C18 column coupled to a Bruker microTOF-Q II. HPLC purification was performed using a Phenomenex C18 Luna $10 \times 250$ $\mathrm{mm}^{2}$ column on Agilent Technologies 1260 Infinity preparative HPLC Ssystem (Wilmington, DE). HPLC purification of ${ }^{18}$ F-labeled compounds was performed on a Varian 
Prostar system (Palo Alto, CA), equipped with a Varian ProStar 325 UV-vis variable wavelength detector and a Bioscan Flow-count in-line radioactivity detector (Washington, DC), all controlled by Galaxie software. The specific radioactivity was calculated as the ratio of the radioactivity eluting at the retention time of product during the preparative HPLC purification to the mass corresponding to the area under the curve of the UV absorption.

\section{Synthesis and Characterization}

(S)-tert-Butyl 6-((tert-Butoxycarbonyl)amino)-2-hydroxyhexanoate 15-(S)-6((tert-Butoxycarbonyl)amino)-2-hydroxyhexanoic acid 14 (250 mg, $1.00 \mathrm{mmol})$ was mixed with tert-butyl-1,3-diisopropylisourea $600 \mathrm{mg}(3.00 \mathrm{mmol})$ in $2 \mathrm{~mL}$ of anhydrous methylene chloride. The reaction was sealed and kept at room temperature for $36 \mathrm{~h}$. (S)-tert-Butyl 6((tert-butoxycarbonyl)amino)-2-hydroxyhexanoate $(135 \mathrm{mg})$ was obtained after silica gel flash column chromatography (hexane/ethyl acetate 2:1 $R_{f}=0.5$ ) (yield $44 \%$ ). ${ }^{1} \mathrm{H}$ NMR (400 MHz, $\left.\mathrm{CDCl}_{3}\right): \delta 4.56(\mathrm{br}, 1 \mathrm{H}), 4.06(\mathrm{dd}, \mathrm{J} 1=7.2 \mathrm{~Hz}, \mathrm{~J} 2=4.4 \mathrm{~Hz}, 1 \mathrm{H}), 3.14(\mathrm{~m}, 2 \mathrm{H})$, 2.25 (br, $1 \mathrm{H}), 1.83-1.75(\mathrm{~m}, 1 \mathrm{H}), 1.65-1.35(\mathrm{~m}, 23 \mathrm{H}) .{ }^{13} \mathrm{C} \mathrm{NMR}\left(125 \mathrm{MHz}, \mathrm{CDCl}_{3}\right): \delta$ 174.6, 156.1, 82.5, 79.2, 70.4, 40.6, 34.0, 29.8, 28.5, 28.1, 22.0. MS: calculated for $\left[\mathrm{C}_{15} \mathrm{H}_{30} \mathrm{NO}\right]^{+}, 304.2118[\mathrm{M}+\mathrm{H}]^{+}$; found 304.2147.

(S)-1-(tert-Butoxy)-6-((tert-butoxycarbonyl)amino)-1-oxohexan-2-yl-1Himidazole-1-carboxylate 16-(S)-tert-Butyl 6-((tert-butoxycarbonyl)amino)-2hydroxyhexanoate 15 (131 mg, $0.43 \mathrm{mmol})$ and 1,1'-carbonyldiimidazole (105 mg, 0.65 mmol) were mixed in $5 \mathrm{~mL}$ of anhydrous methylene chloride. The reaction was kept at room temperature for $1 \mathrm{~h}$. (S)-1-(tert-Butoxy)-6-((tert-butoxycarbonyl)amino)-1-oxohexan-2yl-1 $H$-imidazole-1-carboxylate $(113 \mathrm{mg})$ was obtained by silica gel flash column chromatography (hexane/ethyl acetate 1:2, $R_{f}=0.6$; yield $\left.81 \%\right) .{ }^{1} \mathrm{H}$ NMR $(400 \mathrm{MHz}$, $\left.\mathrm{CDCl}_{3}\right): \delta 8.19(\mathrm{~s}, 1 \mathrm{H}), 7.50(\mathrm{~s}, 1 \mathrm{H}), 7.18(\mathrm{~s}, 1 \mathrm{H}), 5.14(\mathrm{~m}, 1 \mathrm{H}), 4.61(\mathrm{br}, 1 \mathrm{H}), 3.21-3.16(\mathrm{~m}$, $2 \mathrm{H}), 2.05-1.96(\mathrm{~m}, 2 \mathrm{H}), 1.60-1.42(\mathrm{~m}, 22 \mathrm{H}) .{ }^{13} \mathrm{C} \mathrm{NMR}(125 \mathrm{MHz}, \mathrm{CDCl} 3): \delta 167.8,156.0$, 148.3, 137.2, 130.9, 117.2, 83.3, 79.3, 75.8, 40.2, 30.6, 29.7, 28.4, 28.0, 22.4. MS: calculated for $\left[\mathrm{C}_{19} \mathrm{H}_{32} \mathrm{~N}_{3} \mathrm{O}_{6}\right]^{+}, 398.2286[\mathrm{M}+\mathrm{H}]^{+}$; found 398.2309.

(10S,14S)-Tri-tert-butyl 2,2-Dimethyl-4,12-dioxo-3,11-dioxa-5,13diazahexadecane-10,14,16-tricarboxylate 17-(S)-1-(tert-Butoxy)-6-((tertbutoxycarbonyl)amino)-1-oxohexan-2-yl-1 H-imidazole-1-carboxylate $\mathbf{1 6}$ (139 mg, 0.35 $\mathrm{mmol})$ and L-glutamic acid di-tert-butyl ester $(170 \mathrm{mg}, 0.66 \mathrm{mmol})$ were mixed neat. The reaction was kept at $45^{\circ} \mathrm{C}$ for 2 days. (10S,14S)-Tri-tert-butyl 2,2-dimethyl-4,12dioxo-3,11-dioxa-5,13-diazahexadecane-10,14,16-tricarboxylate $(210 \mathrm{mg})$ was obtained by flash column chromatography (hexane/ethyl acetate $2: 1, R_{f}=0.6$; yield $100 \%$ ). ${ }^{1} \mathrm{H}$ NMR (400 MHz, $\left.\mathrm{CDCl}_{3}\right): \delta 5.5(\mathrm{br}, 1 \mathrm{H}), 4.88-4.70(\mathrm{~m}, 1 \mathrm{H}), 4.59(\mathrm{br}, 1 \mathrm{H}), 4.32-4.23(\mathrm{~m}, 1 \mathrm{H})$, 3.16-3.08 (m, 2H), 2.45-2.28 (m, 2H), 2.25-2.10 (m, 1H), 1.95-1.76 (m, 3H), 1.60-1.42 (m, 22H). ${ }^{13} \mathrm{C}$ NMR $(125 \mathrm{MHz}, \mathrm{CDCl} 3) \delta 172.1,171.1,169.9,156.0,155.4,82.4,81.9$, 80.6, 79.1, 73.1, 53.8, 40.4, 31.4, 30.9, 29.6, 28.4, 28.1, 28.0, 22.4. MS: calculated for $\left[\mathrm{C}_{29} \mathrm{H}_{52} \mathrm{~N}_{2} \mathrm{NaO}_{10}\right]^{+}, 611.3514[\mathrm{M}+\mathrm{Na}]^{+}$; found 611.3502 . 
(S)-2-((((S)-5-Amino-1-carboxypentyl)oxy)carbonyl)amino)-pentanedioic Acid Trifluoroacetic Acid Salt, 18-(10S,14S)-Tri-tert-butyl 2,2-dimethyl-4,12-dioxo-3,11dioxa-5,13-diazahexadecane-10,14,16-tricarboxylate (17) $(237 \mathrm{mg})$ was treated with a $6 \mathrm{~mL}$ solution of TFA/methylene chloride (1/1) for $2 \mathrm{~h}$. The final product was purified by HPLC giving $90 \mathrm{mg}$, yield 70\%. ${ }^{1} \mathrm{H}$ NMR (400 MHz, $\mathrm{D}_{2} \mathrm{O} / \mathrm{CD}_{3} \mathrm{CN}$ 1:1): $\delta 4.80(\mathrm{~m}, 1 \mathrm{H}), 4.20-4.11$ (m, 1H, two isomers), 2.90 (t, $J=6.8 \mathrm{~Hz}, 2 \mathrm{H}), 2.43(\mathrm{t}, J=6.8 \mathrm{~Hz}, 2 \mathrm{H}), 2.16-2.09(\mathrm{~m}, 1 \mathrm{H})$, 1.91-1.83 (m, 3H), 1.61-1.58 (m, 2H), 1.43-1.37 (m, 2H). ${ }^{13} \mathrm{C}$ NMR (125 MHz, $\mathrm{D}_{2} \mathrm{O} /$ $\mathrm{CD}_{3} \mathrm{CN} 1: 1$ ): $\delta$ 177.1, 175.7/175.5 (two isomers), 163.1/162.8 (two isomers), 157.3, 73.4, 53.4, 39.2, 36.5, 30.0, 26.2, 26.0, 21.4. MS: calculated for $\left[\mathrm{C}_{12} \mathrm{H}_{21} \mathrm{~N}_{2} \mathrm{O}_{8}\right]^{+}, 321.1292[\mathrm{M}+$ $\mathrm{H}]^{+}$; found 321.1310. HPLC $(10 \mathrm{~mm} \times 250 \mathrm{~mm}$ Phenomenix Luna C18 column, mobile phase 100/0/0.1\% water/acetonitrile/TFA, flow $10 \mathrm{~mL} / \mathrm{min}) .18$ eluted at $14.5 \mathrm{~min}$.

(S)-2-((((S)-1-Carboxy-5-(4-fluorobenzamido)pentyl)oxy)carbonyl)amino)pentanedioic Acid 12- $(S)-2-((((S)-5-$ Amino-1-

carboxypentyl)oxy)carbonyl)amino)pentanedioic acid TFA salt 18 (10 mg, $0.024 \mathrm{mmol})$ was dissolved in $1 \mathrm{~mL}$ of DMSO and $20 \mu \mathrm{L}$ of triethylamine. To the solution, $N$-succinimidyl 4fluorobenzoate ( $11.5 \mathrm{mg}, 0.045 \mathrm{mmol}$ ) was added. The resulting solution was kept at room temperature for $2 \mathrm{~h}$. After the solvent was removed under vacuum and HPLC purification, $12(8 \mathrm{mg})$ was obtained. Yield is 75\%. HPLC (10 mm $\times 250 \mathrm{~mm}$ Phenomenix Luna C18 column, mobile phase 75/25/ $0.1 \%$ water/acetonitrile/TFA, flow $4 \mathrm{~mL} / \mathrm{min}$ ). 12 eluted at 12 min. ${ }^{1} \mathrm{H}$ NMR (400 MHz, $\left.\mathrm{D}_{2} \mathrm{O} / \mathrm{CD}_{3} \mathrm{CN} 1: 1\right): \delta 8.11(\mathrm{~m}, 2 \mathrm{H}), 7.51(\mathrm{t}, J=8.8 \mathrm{~Hz}, 2 \mathrm{H}), 5.16$ $(\mathrm{m}, 1 \mathrm{H}), 4.52-4.45(\mathrm{~m}, 1 \mathrm{H}$, two rotamers), $3.64(\mathrm{t}, J=7.2 \mathrm{~Hz}, 2 \mathrm{H}), 2.74(\mathrm{t}, J=7.2 \mathrm{~Hz}, 2 \mathrm{H})$, 2.46-2.41(m, 1H), 2.22-2.12 (m, 3H), $1.91-1.80(\mathrm{~m}, 2 \mathrm{H}), 1.77-1.68(\mathrm{~m}, 2 \mathrm{H}) .{ }^{13} \mathrm{C}$ NMR (125 MHz, $\left.\mathrm{D}_{2} \mathrm{O} / \mathrm{CD}_{3} \mathrm{CN} 1: 1\right): \delta 176.3,174.8,174.2,168.4,166.0 / 164.0$ (coupled with F), 156.9, 130.9, 130.2/130.1 (coupled with F), 116.0/115.9 (coupled with F), 73.2, 53.6, 39.9, $30.9,30.3,28.7,26.7,22.5$. MS: calculated for $\left[\mathrm{C}_{19} \mathrm{H}_{23} \mathrm{FN}_{2} \mathrm{NaO}_{9}\right]^{+}, 465.1280[\mathrm{M}+\mathrm{Na}]^{+}$; found 465.1295 .

(S)-Dimethyl 2-((1H-Imidazole-1-carbonyl)oxy)-pentanedioate 20-(S)-Dimethyl 2-hydroxypentanedioate 19 (220 mg, $1.25 \mathrm{mmol})$ and 1,1'-carbonyldiimidazole (300 mg, $1.85 \mathrm{mmol}$ ) were mixed in $5 \mathrm{~mL}$ of anhydrous methylene chloride. The reaction was kept at room temperature for $1 \mathrm{~h}$. (S)-Dimethyl 2-((1H-imidazole-1-carbonyl)oxy)pentanedioate (290 mg was obtained by silica gel flash column chromatography (hexane/ethyl acetate 1:2, $\left.R_{f}=0.4\right)$. The yield is $85 \% .{ }^{1} \mathrm{H}$ NMR $\left(400 \mathrm{MHz}, \mathrm{CDCl}_{3}\right): \delta 8.20(\mathrm{~s}, 1 \mathrm{H}), 7.49(\mathrm{~s}, 1 \mathrm{H}), 7.12$ $(\mathrm{s}, 1 \mathrm{H}), 5.35(\mathrm{dd}, \mathrm{J} 1=8.0 \mathrm{~Hz}, \mathrm{~J} 2=4.8 \mathrm{~Hz}, 1 \mathrm{H}), 3.83(\mathrm{~s}, 3 \mathrm{H}), 3.72(\mathrm{~s}, 3 \mathrm{H}), 2.58-2.34(\mathrm{~m}$, 4H). ${ }^{13} \mathrm{C} \mathrm{NMR}\left(125 \mathrm{MHz}, \mathrm{CDCl}_{3}\right) \delta 172.3,168.7,148.0,137.2,131.0,117.2,74.4,53.0$, 29.4, 26.1. MS: calculated for $\left[\mathrm{C}_{11} \mathrm{H}_{15} \mathrm{~N}_{2} \mathrm{O}_{6}\right]^{+}, 271.0925[\mathrm{M}+\mathrm{H}]^{+}$; found 271.0932.

(10S,14S)-10-tert-Butyl 14,16-Dimethyl 2,2-dimethyl-4,12-dioxo-3,13dioxa-5,11-diazahexadecane-10,14,16-tricarboxylate 21-( $S)$-Dimethyl 2-((1Himidazole-1-carbonyl)oxy)-pentanedioate 20 (110 mg, $0.41 \mathrm{mmol})$ was dissolved in $2 \mathrm{~mL}$ of $\mathrm{MeCN}$ and $1 \mathrm{~mL}$ of MeI. The mixture was sealed and heated at $55^{\circ} \mathrm{C}$ for $3 \mathrm{~h}$. The solvent was removed under vacuum. Then, a mixture of $N^{E}$-Boc-lysine-tert-butyl ester hydrochloride $(275 \mathrm{mg}, 0.81 \mathrm{mmol})$ and triethylamine $(0.5 \mathrm{~mL})$ in $5 \mathrm{~mL}$ of DMSO was added. The reaction was kept at room temperature overnight. (10S,14S)-10-tert-Butyl-14,16-dimethyl 
2,2-dimethyl-4,12-dioxo-3,13-dioxa-5,11-diazahexade-cane-10,14,16-tricarboxylate was obtained by flash column chromatography (hexane/ethyl acetate 1:2, $R_{f}=0.5$ ). The yield was $150 \mathrm{mg}(75 \%) .{ }^{1} \mathrm{H}$ NMR (400 MHz, $\left.\mathrm{CDCl}_{3}\right) \delta 5.49(\mathrm{br}, 1 \mathrm{H}), 5.08-5.06(\mathrm{~m}, 1 \mathrm{H}), 4.70$ (br, $1 \mathrm{H}), 3.80(\mathrm{~s}, 3 \mathrm{H}), 3.73(\mathrm{~s}, 3 \mathrm{H}) 3.16-3.13(\mathrm{~m}, 2 \mathrm{H}), 2.54-2.48(\mathrm{~m}, 2 \mathrm{H}), 2.28-2.17(\mathrm{~m}$, $2 \mathrm{H}), 1.89-1.86(\mathrm{~m}, 1 \mathrm{H}), 1.68-1.64(\mathrm{~m}, 1 \mathrm{H}), 1.63-1.41(\mathrm{~m}, 22 \mathrm{H}) .{ }^{13} \mathrm{C} \mathrm{NMR}(125 \mathrm{MHz}$, $\left.\mathrm{CDCl}_{3}\right) \delta 172.8,171.3,170.8,156.1,154.9,82.3,79.1,74.6,71.6,54.3,52.4,51.8,40.2$, 32.5, 29.5, 28.5, 28.0, 26.5, 22.2. MS: calculated for $\left[\mathrm{C}_{23} \mathrm{H}_{41} \mathrm{~N}_{2} \mathrm{O}_{10}\right]^{+}, 505.2756[\mathrm{M}+\mathrm{H}]^{+}$; found 505.2735 .

(S)-2-(((S)-5-Amino-1-carboxypentyl)carbamoyl)oxy)-pentanedioic Acid 22(10S,14S)-10-tert-Butyl 14,16-dimethyl 2,2-dimethyl-4,12-dioxo-3,13-dioxa-5,11diazahexadecane-10,14,16-tricar-boxylate $(\mathbf{2 1})(150 \mathrm{mg}, 0.30 \mathrm{mmol})$ was treated with a 6 $\mathrm{mL}$ solution of TFA/methylene chloride (1/1) for $2 \mathrm{~h}$. The solvent was removed under vacuum, and the remaining material was redissolved in $5 \mathrm{~mL}$ of $\mathrm{THF} / \mathrm{H}_{2} \mathrm{O}(1 / 1)$. To the solution, $\mathrm{LiOH}$ monohydrate $(40 \mathrm{mg}, 1 \mathrm{mmol})$ was added, and the reaction was stirred at room temperature for $4 \mathrm{~h}$. The final product was purified by HPLC to provide $80 \mathrm{mg}$, yield 65\%. ${ }^{1} \mathrm{H}$ NMR (400 MHz, $\left.\mathrm{D}_{2} \mathrm{O}\right): \delta 4.82-4.77(\mathrm{~m}, 1 \mathrm{H}), 4.11-4.07(\mathrm{~m}, 1 \mathrm{H}), 2.90$ (t, $J=7.2$ $\mathrm{Hz}, 2 \mathrm{H}), 2.62-2.42(\mathrm{~m}, 2 \mathrm{H}), 2.14-2.00(\mathrm{~m}, 2 \mathrm{H}), 1.87-1.80(\mathrm{~m}, 1 \mathrm{H}), 1.70-1.60(\mathrm{~m}, 3 \mathrm{H})$, 1.41-1.35 (m, 2H). ${ }^{13} \mathrm{C}$ NMR (125 MHz, $\left.\mathrm{D}_{2} \mathrm{O}\right): \delta 177.1,175.9,174.6,157.1,72.3,53.7$, 39.2, 30.2, 29.3, 26.1, 21.9. MS: calculated for $\left[\mathrm{C}_{12} \mathrm{H}_{21} \mathrm{~N}_{2} \mathrm{O}_{8}\right]^{+}, 321.1292[\mathrm{M}+\mathrm{H}]^{+}$; found 321.1318. HPLC $(10 \mathrm{~mm} \times 250 \mathrm{~mm}$ Phenomenix Luna C18 column, mobile phase 97/3/0.1\% water/ acetonitrile/TFA, flow $10 \mathrm{~mL} / \mathrm{min}) .22$ eluted at $8 \mathrm{~min}$.

\section{(S)-2-((((S)-1-Carboxy-5-(4-fluorobenzamido)pentyl)-} carbamoyl)oxy)pentanedioic Acid 13- $(S)$-2-((( $S)$-5-Amino-1-

carboxypentyl)carbamoyl)oxy)pentanedioic acid TFA salt 22 (10 mg, $0.024 \mathrm{mmol})$ was dissolved in $1 \mathrm{~mL}$ of DMSO and $20 \mu \mathrm{L}$ of triethylamine. To the solution, $N$-succinimidyl 4fluorobenzoate $(11.5 \mathrm{mg}, 0.045 \mathrm{mmol})$ was added. The resulting solution was kept at room temperature for $2 \mathrm{~h}$. After solvent was removed under vacuum, $9 \mathrm{mg}$ of product was obtained after HPLC purification. The yield was $89 \% .{ }^{1} \mathrm{H}$ NMR $\left(500 \mathrm{MHz}, \mathrm{D}_{2} \mathrm{O} / \mathrm{CD}_{3} \mathrm{CN}\right.$ 1:1): $87.77-7.73(\mathrm{~m}, 2 \mathrm{H}), 7.17-7.13(\mathrm{~m}, 2 \mathrm{H}), 4.82-4.80(\mathrm{~m}, 1 \mathrm{H}), 4.06-4.04(\mathrm{~m}, 1 \mathrm{H}), 3.29-$ $3.26(\mathrm{~m}, 2 \mathrm{H}), 2.42-2.36(\mathrm{~m}, 2 \mathrm{H}), 2.10-2.06(\mathrm{~m}, 1 \mathrm{H}), 2.00-1.95(\mathrm{~m}, 1 \mathrm{H}), 1.80-1.76(\mathrm{~m}, 1 \mathrm{H})$, $1.67-1.63(\mathrm{~m}, 1 \mathrm{H}), 1.54(\mathrm{~m}, 2 \mathrm{H}), 1.38(\mathrm{~m}, 2 \mathrm{H}) .{ }^{13} \mathrm{C} \mathrm{NMR}\left(125 \mathrm{MHz}, \mathrm{D}_{2} \mathrm{O} \mathrm{CD}_{3} \mathrm{CN} 1: 1\right): \delta$ 178.4, 177.8, 175.8, 170.6, 168.3/166.4 (coupled with F), 159.1, 133.4, 132.6/132.5 (coupled with F), 118.3/118.2 (coupled with F), 74.5, 56.7, 42.2, 33.5, 32.0, 31.0, 28.9, 25.4. MS: calculated for $\left[\mathrm{C}_{19} \mathrm{H}_{23} \mathrm{FN}_{2} \mathrm{NaO}_{9}\right]^{+}, 465.1280[\mathrm{M}+\mathrm{Na}]^{+}$; found 465.1306. HPLC (10 $\mathrm{mm} \times 250 \mathrm{~mm}$ Phenomenix Luna C18 column, mobile phase 75/25/0.1\% water/acetonitrile/ TFA, flow $4 \mathrm{~mL} / \mathrm{min}) .13$ eluted at $11.5 \mathrm{~min}$.

\section{(S)-2-((((S)-5-(4-Bromo-2-fluorobenzamido)-1-} carboxypentyl)carbamoyl)oxy)pentanedioic Acid 23- $(S)-2-(((S)-5$-Amino-1carboxypentyl)carbamoyl)oxy)pentanedioic acid TFA salt $22(10 \mathrm{mg}, 0.024 \mathrm{mmol})$ was dissolved in $1 \mathrm{~mL}$ of DMSO and $20 \mu \mathrm{L}$ of triethylamine. To the solution, $N$-succinimidyl 4bromo-2-fluorobenzoate (14 mg, $0.045 \mathrm{mmol}$ ) was added. The resulting solution was kept at room temperature for $2 \mathrm{~h}$. After removal of the solvent under vacuum and HPLC 
purification, $8 \mathrm{mg}$ of product was obtained. The yield was $64 \% .{ }^{1} \mathrm{H}$ NMR $(500 \mathrm{MHz}$, $\left.\mathrm{D}_{2} \mathrm{O} / \mathrm{CD}_{3} \mathrm{CN} 1: 1\right): \delta 7.55(\mathrm{t}, J=8.2 \mathrm{~Hz}, 1 \mathrm{H}), 7.43-7.41(\mathrm{~m}, 2 \mathrm{H}), 4.82-4.80(\mathrm{~m}, 1 \mathrm{H}), 4.06-$ $4.03(\mathrm{~m}, 1 \mathrm{H}), 3.29(\mathrm{t}, J=6.6 \mathrm{~Hz}, 2 \mathrm{H}), 2.43-2.36(\mathrm{~m}, 2 \mathrm{H}), 2.10-2.05(\mathrm{~m}, 1 \mathrm{H}), 2.01-1.95(\mathrm{~m}$, $1 \mathrm{H}), 1.85-1.76(\mathrm{~m}, 1 \mathrm{H}), 1.67-1.63(\mathrm{~m}, 1 \mathrm{H}), 1.55-1.50(\mathrm{~m}, 2 \mathrm{H}), 1.40-1.36(\mathrm{~m}, 2 \mathrm{H}) .{ }^{13} \mathrm{C}$ NMR (125 MHz, $\left.\mathrm{D}_{2} \mathrm{O} / \mathrm{CD}_{3} \mathrm{CN} 1: 1\right): \delta 176.2,175.5,173.6,165.1,160.9 / 158.9$ (coupled with F), 156.7, 132.1, 128.5, 125.8, 122.3/122.2 (coupled with F), 120.3/120.1 (coupled with F), 72.2, 54.3, 39.9, 31.1, 29.7, 28.5, 26.5, 22.9. MS: calculated for $\left[\mathrm{C}_{19} \mathrm{H}_{22} \mathrm{BrFN}_{2} \mathrm{NaO}_{9}\right]^{+}$, $543.0385[\mathrm{M}+\mathrm{Na}]^{+}$; found 543.0372. HPLC $(10 \mathrm{~mm} \times 250 \mathrm{~mm}$ Phenomenix Luna C18 column, mobile phase 70/30/0.1\% water/ acetonitrile/TFA, flow $4 \mathrm{~mL} / \mathrm{min}$ ). 23 eluted at 12.9 min.

\section{(S)-2-((((S)-1-Carboxy-5-(2-fluoro-4-iodobenzamidopentyl)-} carbamoyl)oxy)pentanedioic Acid 24-(S)-2-((((S)-5-Amino-1carboxypentyl)carbamoyl)oxy)pentanedioic acid TFA salt 22 (10 mg, $0.024 \mathrm{mmol})$ was dissolved in $1 \mathrm{~mL}$ of DMSO and $20 \mu \mathrm{L}$ of triethylamine. To the solution, $N$-succinimidyl 4iodo-2-fluoroben-zoate ( $15 \mathrm{mg}, 0.045 \mathrm{mmol}$ ) was added. The resulting solution was kept at room temperature for $2 \mathrm{~h}$. After removal of solvent under vacuum and HPLC purification, 7 mg was obtained. The yield was 51\%. ${ }^{1} \mathrm{H}$ NMR $\left(500 \mathrm{MHz}, \mathrm{D}_{2} \mathrm{O} / \mathrm{CD}_{3} \mathrm{CN} 1: 1\right): \delta 7.63-7.59$ $(\mathrm{m}, 2 \mathrm{H}), 7.39(\mathrm{t}, J=8.0 \mathrm{~Hz}, 1 \mathrm{H}), 4.82-4.79(\mathrm{~m}, 1 \mathrm{H}), 4.06-4.03(\mathrm{~m}, 1 \mathrm{H}), 3.28(\mathrm{t}, J=6.8 \mathrm{~Hz}$, $2 \mathrm{H}), 2.46-2.34(\mathrm{~m}, 2 \mathrm{H}), 2.12-2.05(\mathrm{~m}, 1 \mathrm{H}), 2.01-1.95(\mathrm{~m}, 1 \mathrm{H}), 1.82-1.75(\mathrm{~m}, 1 \mathrm{H}), 1.69-$ $1.61(\mathrm{~m}, 1 \mathrm{H}), 1.58-1.48(\mathrm{~m}, 2 \mathrm{H}), 1.42-1.34(\mathrm{~m}, 2 \mathrm{H}) .{ }^{13} \mathrm{C} \mathrm{NMR}\left(125 \mathrm{MHz}, \mathrm{D}_{2} \mathrm{O} / \mathrm{CD}_{3} \mathrm{CN}\right.$ 1:1): $\delta$ 176.2, 175.5, 173.5, 165.2, 160.6/158.6 (coupled with F), 156.8, 134.6, 132.1, 126.1/125.9 (coupled with F), 123.0/122.9 (coupled with F), 97.5/97.4 (coupled with F), 72.2, 54.4, 40.0, 31.2, 29.7, 28.7, 26.6, 23.0. MS: calculated for $\left[\mathrm{C}_{19} \mathrm{H}_{23} \mathrm{IFN}_{2} \mathrm{O}_{9}\right]^{+}, 569.0427$ $[\mathrm{M}+\mathrm{H}]^{+}$; found 569.0438. HPLC $(10 \mathrm{~mm} \times 250 \mathrm{~mm}$ Phenomenix Luna C18 column, mobile phase $70 / 30 / 0.1 \%$ water/acetonitrile/TFA, flow $4 \mathrm{~mL} / \mathrm{min}$ ). 24 eluted at $17.0 \mathrm{~min}$.

\section{(S)-2-(3-((S)-5-(4-Bromo-2-fluorobenzamido)-1-} carboxypentyl)ureido)pentanedioic Acid 26-(S)-2-(3-((S)-5-Amino-1-

carboxypentyl)ureido)pentanedioic acid TFA salt 25 (10 mg, $0.024 \mathrm{mmol})$ was dissolved in $1 \mathrm{~mL}$ of DMSO and $20 \mu \mathrm{L}$ of triethylamine. To the solution, $N$-succinimidyl 4-bromo-2fluorobenzoate (14 $\mathrm{mg}, 0.045 \mathrm{mmol}$ ) was added. The resulting solution was kept at room temperature for $2 \mathrm{~h}$. After the solvent was removed under vacuum, $9 \mathrm{mg}$ was obtained after HPLC purification. The yield was $71 \% .{ }^{1} \mathrm{H}$ NMR $\left(500 \mathrm{MHz}, \mathrm{D}_{2} \mathrm{O} / \mathrm{CD}_{3} \mathrm{CN} 1: 1\right): \delta 7.58-7.54$ $(\mathrm{m}, 1 \mathrm{H}), 7.46-7.40(\mathrm{~m}, 2 \mathrm{H}), 4.16-4.09(\mathrm{~m}, 2 \mathrm{H}), 3.28(\mathrm{t}, J=6.8 \mathrm{~Hz}, 2 \mathrm{H}), 2.35(\mathrm{t}, J=7.5 \mathrm{~Hz}$, $2 \mathrm{H}), 2.06-1.99(\mathrm{~m}, 1 \mathrm{H}), 1.85-1.71(\mathrm{~m}, 2 \mathrm{H}), 1.66-1.50(\mathrm{~m}, 3 \mathrm{H}), 1.40-1.34(\mathrm{~m}, 2 \mathrm{H}) .{ }^{13} \mathrm{C}$ NMR (125 MHz, $\left.\mathrm{D}_{2} \mathrm{O} / \mathrm{CD}_{3} \mathrm{CN} 1: 1\right): \delta 176.6,176.5,175.7,165.2,160.9 / 158.9$ (coupled with F), 159.0, 132.0, 128.5, 125.8/125.7 (coupled with F), 122.3/122.2 (coupled with F), 120.3/120.1 (coupled with F), 53.4, 52.7, 39.9, 31.4, 30.4, 28.6, 27.1, 22.9. MS: calculated for $\left[\mathrm{C}_{19} \mathrm{H}_{24} \mathrm{BrFN}_{3} \mathrm{O}_{8}\right]^{+}, 520.0725[\mathrm{M}+\mathrm{H}]^{+}$; found 520.0762. HPLC $(10 \mathrm{~mm} \times 250 \mathrm{~mm}$ Phenomenix Luna C18 column, mobile phase 75/25/0.1\% water/acetonitrile/TFA, flow 10 $\mathrm{mL} / \mathrm{min}) .26$ eluted at $7.2 \mathrm{~min}$.

(S)-2-(3-((S)-5-(4-lodo-2-fluorobenzamido)-1carboxypentyl)ureido)pentanedioic Acid 27-(S)-2-(3-((S)-5-Amino-1- 
carboxypentyl)ureido)pentanedioic acid TFA salt $25(10 \mathrm{mg}, 0.031 \mathrm{mmol})$ was dissolved in $1 \mathrm{~mL}$ of DMSO and $20 \mu \mathrm{L}$ of triethylamine. To the solution, $N$-succinimidyl 4-iodo-2fluorobenzoate (14 mg, $0.045 \mathrm{mmol}$ ) was added. The resulting solution was kept at room temperature for $2 \mathrm{~h}$. After the solvent was removed under vacuum, $11 \mathrm{mg}$ of 27 was obtained after HPLC purification. The yield was $81 \% .{ }^{1} \mathrm{H}$ NMR $\left(500 \mathrm{MHz}, \mathrm{D}_{2} \mathrm{O} / \mathrm{CD}_{3} \mathrm{CN} 1: 1\right): \delta$, 7.63-7.60 (m, 2H), 7.38 (t, $J=7.8 \mathrm{~Hz}, 1 \mathrm{H}), 4.14-4.07(\mathrm{~m}, 2 \mathrm{H}), 3.27$ (t, $J=6.9 \mathrm{~Hz}, 2 \mathrm{H})$, $2.34(\mathrm{t}, J=7.6 \mathrm{~Hz}, 2 \mathrm{H}), 2.05-1.96(\mathrm{~m}, 1 \mathrm{H}), 1.85-1.72(\mathrm{~m}, 2 \mathrm{H}), 1.65-1.49(\mathrm{~m}, 3 \mathrm{H}), 1.38-$ 1.33 (m, 2H). ${ }^{13} \mathrm{C}$ NMR (125 MHz, $\left.\mathrm{D}_{2} \mathrm{O} / \mathrm{CD}_{3} \mathrm{CN} 1: 1\right): \delta 176.7,176.0,165.2,160.5 / 158.5$ (coupled with F), 159.1, 134.6, 132.0, 126.1/125.9 (coupled with F), 122.9/122.8 (coupled with F), 97.5, 53.6, 53.0, 40.0, 31.6, 30.5, 28.7, 27.4, 23.0. MS: calculated for $\left[\mathrm{C}_{19} \mathrm{H}_{23} \mathrm{IFN}_{3} \mathrm{NaO}_{8}\right]^{+}, 590.0406[\mathrm{M}+\mathrm{Na}]^{+}$; found 590.0406. HPLC $(10 \mathrm{~mm} \times 250 \mathrm{~mm}$ Phenomenix Luna C18 column, mobile phase 75/25/0.1\% water/acetonitrile/TFA, flow 10 $\mathrm{mL} / \mathrm{min}) .27$ eluted at $8.5 \mathrm{~min}$.

\section{NAALADase Assay}

PSMA inhibitory activity was determined using a modification of the fluorescence-based Amplex red glutamic acid assay (Life Technologies, Grand Island, NY) ${ }^{10 \mathrm{~b}}$ Briefly, lysates of LNCaP cell extracts $(25 \mu \mathrm{L})$ were incubated with the inhibitor $(12.5 \mu \mathrm{L})$ in the presence of $4 \mu \mathrm{M} N$-acetylaspartylglutamate (NAAG) $(12.5 \mu \mathrm{L})$ for $120 \mathrm{~min}$. The amount of the glutamate released by NAAG hydrolysis was measured by incubating with a working solution $(50 \mu \mathrm{L})$ of the Amplex red glutamic acid kit for $60 \mathrm{~min}$. Fluorescence was measured with a VICTOR3V multilabel plate reader (PerkinElmer Inc., Waltham, MA) with excitation at $490 \mathrm{~nm}$ and emission at $642 \mathrm{~nm}$. Inhibition curves were determined using semilog plots, and $\mathrm{IC}_{50}$ values were determined at the concentration at which enzyme activity was inhibited by $50 \%$. Enzyme inhibitory constants ( $K_{\mathrm{i}}$ values) were generated using the ChengPrusoff conversion. ${ }^{31}$ Assays were performed in triplicate. Data analysis was performed using GraphPad Prism, version 4.00, for Windows (GraphPad Software, San Diego, CA).

\section{Cell Lines and Mouse Models}

Sublines of the androgen-independent PC3 human prostate cancer cell line, originally derived from an advanced androgen independent bone metastasis, were used. Those sublines have been modified to express high levels of PSMA (PSMA+ PC3 PIP) or are devoid of PSMA expression (PSMA- PC3 flu) and were generously provided by Dr. Warren Heston (Cleveland Clinic). Prostate cancer cell lines were grown in RPMI 1640 medium (Corning Cellgro, Manassas, VA) containing 10\% fetal bovine serum (FBS) (Sigma-Aldrich, St.Louis, MO) and $1 \%$ penicillin-streptomycin (Corning Cellgro, Manassas, VA). PSMA+ PC3 PIP cells were grown under $20 \mu \mathrm{g} / \mathrm{mL}$ of puromycin selection in the growth medium to maintain PSMA expression. All cell cultures were maintained in an atmosphere containing $5 \%$ carbon dioxide $\left(\mathrm{CO}_{2}\right)$ at $37.0^{\circ} \mathrm{C}$ in a humidified incubator.

Animal studies were carried out in full compliance with the regulations of the Johns Hopkins Animal Care and Use Committee. Six- to eight-week-old male, nonobese diabetic (NOD)/severe-combined immunodeficient (SCID) mice (Johns Hopkins Immune Compromised Core) were implanted subcutaneously with PSMA+ PC3 PIP and PSMAPC3 flu cells $\left(1 \times 10^{6}\right.$ in $100 \mu \mathrm{L}$ of HBSS (Corning Cellgro, Manassas, VA) at the forward 
right and left flanks, respectively. Mice were imaged or used in biodistribution assays when the xenografts reached 5-7 $\mathrm{mm}$ in diameter.

\section{Radiosynthesis}

${ }^{18}$ F-Fluoride was produced by a General Electric PETtrace biomedical cyclotron (GE HealthCare, Wauwatsosa, WI) using $18 \mathrm{MeV}$ proton bombardment on an $\left[{ }^{18} \mathrm{O}\right] \mathrm{H}_{2} \mathrm{O}$ target and trapped on a Chromafix 30-PS-HCO3 QMA cartridge. The cartridge was eluted with 0.5 $\mathrm{mL}$ of a solution of potassium bicarbonate $(4.5 \mathrm{mg} / 0.5 \mathrm{~mL})$ into a $3 \mathrm{~mL}$ Wheaton reaction vial. To this was added 15-18 mg of 4,7,13,16,21,24-hexaoxa-1,10-diazabicyclo[8.8.8]hexacosane $\left(\mathrm{K}_{2.2 .2}\right)$ in $1 \mathrm{~mL}$ of acetonitrile and heated to $100{ }^{\circ} \mathrm{C}$ under a stream of argon gas to dryness. Further drying was accomplished by azeotropic distillation using $3 \times 0.5 \mathrm{~mL}$ additions of acetonitrile. The vial was cooled to room temperature, and a solution of 8-16 $\mathrm{mg}$ of the 4-halo-2-nitrobenzaldehyde in $250 \mu \mathrm{L}$ of DMSO was added and heated at $120^{\circ} \mathrm{C}$ for $20 \mathrm{~min}$ and then cooled to room temperature and diluted to a volume of $2 \mathrm{~mL}$ with $25 \%$ acetonitrile/water for purification by radio-HPLC $(10 \mathrm{~mm} \times 250 \mathrm{~mm}$ Phenomenex Luna C18 column, 54/46/0.1 water/acetonitrile/TFA, $4 \mathrm{~mL} / \mathrm{min})$. 4-Bromo-2- $\left[{ }^{18} \mathrm{~F}\right]$ fluorobenzaldehyde eluted at $18.5 \mathrm{~min}$, and 4-iodo-2-[ $\left[{ }^{18} \mathrm{~F}\right]$ fluorobenzaldehyde eluted at $23 \mathrm{~min}$. The product HPLC fraction was diluted with water to a volume of $45 \mathrm{~mL}$ and loaded onto an Oasis HLB Sep-Pak cartridge, washed with $5 \mathrm{~mL}$ of water, then dried by passing argon through the cartridge for $2 \mathrm{~min}$. A sodium sulfate drying tube was then added to the end of the Oasis Sep-Pak, and the aldehyde eluted with $2 \mathrm{~mL}$ of methylene chloride into another Wheaton reaction vial. The methylene chloride is evaporated under a stream of argon, and the residue was dissolved in $0.5 \mathrm{~mL}$ of acetonitrile. To this was added $50 \mathrm{mg}$ of $N$-hydroxysuccinimide and $28 \mathrm{mg}$ of (diacetoxyiodo)benzene. The vial was capped, shaken, and allowed to stand at room temperature for $15 \mathrm{~min}$, then diluted with $1 / 1$ acetonitrile/water for injection onto the radio-HPLC $(10 \mathrm{~mm} \times 250 \mathrm{~mm}$ Phenomenex Luna C18 column, 50/ 50/0.1 water/ acetonitrile/TFA, $4 \mathrm{~mL} / \mathrm{m})$. $N$-Succinimidyl-4-bromo-2- $\left[{ }^{18} \mathrm{~F}\right]$ fluorobenzoate eluted at 12 $\min$, and $\mathrm{N}$-succinimidyl-4-iodo-2-[ $\left[{ }^{18} \mathrm{~F}\right]$ fluorobenzoate eluted at $14 \mathrm{~min}$. The product HPLC fraction was diluted with water to a volume of $45 \mathrm{~mL}$ and loaded onto a Waters C18 Sep-Pak Plus cartridge, washed with $5 \mathrm{~mL}$ of water, then dried by passing argon through it for $2 \mathrm{~min}$. A sodium sulfate drying tube was then added to the end of the C18 Sep-Pak Plus cartridge, and the ester was eluted with $2 \mathrm{~mL}$ of methylene chloride into another Wheaton reaction vial. The methylene chloride was evaporated under a stream of argon. To the residue was added a solution of $3 \mathrm{mg}(S)$-2-((( $S)$-5-amino-1-carboxypentyl)carbamoyl)oxy)pentanedioic acid (22) in $200 \mu \mathrm{L}$ of DMSO and $5 \mu \mathrm{L}$ of diisopropylethylamine. The vial was capped, shaken, and heated at $50{ }^{\circ} \mathrm{C}$ for $10 \mathrm{~min}$ and then allowed to cool to room temperature. The reaction mixture was acidified by the addition of $20 \mu \mathrm{L}$ of TFA and diluted with water for purification by HPLC $(10 \mathrm{~mm} \times 250 \mathrm{~mm}$ Phenomenex Luna C18 column, 70/30/0.1 water/ acetonitrile/TFA, $4 \mathrm{~mL} / \mathrm{m})$. Compound $\left[{ }^{18} \mathrm{~F}\right] \mathbf{2 3}$ eluted at $13 \mathrm{~min}$, and $\left[{ }^{18} \mathrm{~F}\right] \mathbf{2 4}$ eluted at 17 $\min$. The nondecay corrected radiochemical yield from ${ }^{18} \mathrm{~F}$ fluoride was $5 \%$ for $\left[{ }^{18} \mathrm{~F}\right] 35$ and $3 \%$ for $\left[{ }^{18} \mathrm{~F}\right] \mathbf{2 4}$. Specific radioactivity ranged from $37000-177600 \mathrm{GBq} / \mathrm{mmol}(1000-4800$ $\mathrm{Ci} / \mathrm{mmol})$ for $\left[{ }^{18} \mathrm{~F}\right] \mathbf{2 3}$ and from $31080-92500 \mathrm{GBq} / \mathrm{mmol}(840-2500 \mathrm{Ci} / \mathrm{mmol})$ for $\left[{ }^{18} \mathrm{~F}\right] \mathbf{2 4}$. 


\section{Imaging}

Dynamic and whole-body PET and CT images were acquired on an eXplore VISTA smallanimal PET (GE Healthcare) and an X-SPECT small SPECT/CT system (Gamma Medica Ideas), respectively. For imaging studies, anesthesia was induced with $3 \%$ and maintained under $1.5 \%$ isoflurane $(\mathrm{v} / \mathrm{v})$ in oxygen. PET or PET-CT imaging studies were performed on NOD/SCID mice bearing PSMA+ PC-3 PIP and PSMA- PC-3 flu tumors. Following intravenous injection of $\left[{ }^{18} \mathrm{~F}\right] \mathbf{1 2},\left[{ }^{18} \mathrm{~F}\right] \mathbf{1 3},\left[{ }^{18} \mathrm{~F}\right] \mathbf{2 3}$, or $\left[{ }^{18} \mathrm{~F}\right] \mathbf{2 4}$, PET images were acquired at $10 \mathrm{~min}, 30 \mathrm{~min}, 1 \mathrm{~h}, 2 \mathrm{~h}$, and $4 \mathrm{~h}$ pi as a pseudodynamic scan; i.e., a sequence of successive whole-body images were acquired in two bed positions. The dwell time at each bed position was $10 \mathrm{~min}$ for a total scan time of $20 \mathrm{~min}$. An energy window of 250-700 keV was used. Images were reconstructed using the FORE/2D-OSEM method (one iteration, 16 subsets) and included correction for radioactive decay, scanner dead time, and scattered radiation.

\section{Biodistribution}

Mice bearing PSMA+ PC3 PIP and PSMA- PC3 flu xenografts ${ }^{32}$ were injected via the tail vein with $740 \mathrm{kBq}\left(20 \mu \mathrm{C}_{\mathrm{i}}\right)$ of $\left[{ }^{18} \mathrm{~F}\right] \mathbf{1 2},\left[{ }^{18} \mathrm{~F}\right] \mathbf{2 3}$, or $\left[{ }^{18} \mathrm{~F}\right] \mathbf{2 4}$ in $200 \mu \mathrm{L}$ of saline. At various time points postinjection mice were sacrificed by cervical dislocation and the blood was immediately collected by cardiac puncture. The heart, lungs, liver, stomach, pancreas, spleen, fat, kidney, muscle, small and large intestines, urinary bladder, PSMA+ PC3 PIP, and PSMA- PC-3 flu tumors were collected. Each organ was weighed, and the tissue radioactivity was measured with an automated $\gamma$ counter (1282 Compugamma CS, Pharmacia/LKBNuclear, Inc., Mt. Waverly, Victoria, Australia). The \% ID/g was calculated by comparison with samples of a dilution of a standard dose. All measurements were corrected for decay. Data are expressed as the mean \pm standard deviation (SD).

\section{Molecular Modeling}

All molecular modeling experiments were performed using Discovery Studio 4.0 developed by Accelrys, Inc. (San Diego, CA).

Protein and Ligand Structure Preparation-The X-ray structure of PSMA cocrystallized with the competitive inhibitor DCIBzL ${ }^{13 \mathrm{~d}}$ (PDB code $3 \mathrm{D} 7 \mathrm{H}$ ) was downloaded from the Protein Data Bank (RCSB, http://www.rcsb.org/pdb/home/home.do). The water molecules were removed, while the cocrystallized ligand was used as a template to sketch compounds using the Sketch Molecules module in Discovery Studio.

In Situ Ligand Minimization-Each ligand was minimized while binding to its target protein using the in situ ligand minimization module with the following parameters: CHARMM as an input force field, minimization algorithm as smart minimizer, maximum minimization steps equal to 1000 , minimization with rms gradient equal to $0.001 \AA$, and minimization energy change set equal to zero. After the minimization protocol was executed, the in situ minimized ligands were stripped of their nonpolar hydrogens to simplify the overall view. The protein was depicted in the form of a light gray line ribbon. The bound ligand is depicted as a stick with atoms color-coded according to element: carbon (gray), nitrogen (blue), and oxygen (red). 


\section{Supplementary Material}

Refer to Web version on PubMed Central for supplementary material.

\section{Acknowledgments}

This work was supported in part by Avid Radiopharmaceuticals, Grants CA134675, CA183031, CA006973, and CA184228.

\section{REFERENCES}

1. (a) Mesters JR, Barinka C, Li W, Tsukamoto T, Majer P, Slusher BS, Konvalinka J, Hilgenfeld R. Structure of glutamate carboxypeptidase II, a drug target in neuronal damage and prostate cancer. EMBO J. 2006; 25(6):1375-1384. [PubMed: 16467855] (b) Barinka C, Sacha P, Sklenar J, Man P, Bezouska K, Slusher BS, Konvalinka J. Identification of the N-glycosylation sites on glutamate carboxypeptidase II necessary for proteolytic activity. Protein Sci. 2004; 13(6):1627-1635. [PubMed: 15152093] (c) Pinto JT, Suffoletto BP, Berzin TM, Qiao CH, Lin S, Tong WP, May F, Mukherjee B, Heston WD. Prostate-specific membrane antigen: a novel folate hydrolase in human prostatic carcinoma cells. Clin. Cancer Res. 1996; 2(9):1445-1451. [PubMed: 9816319]

2. Ghosh A, Heston WD. Tumor target prostate specific membrane antigen (PSMA) and its regulation in prostate cancer. J. Cell. Biochem. 2004; 91(3):528-539. [PubMed: 14755683]

3. Rajasekaran AK, Anilkumar G, Christiansen JJ. Is prostate-specific membrane antigen a multifunctional protein. Am. J. Physiol.: Cell Physiol. 2005; 288(5):C975-C981. [PubMed: 15840561]

4. Chang SS, Reuter VE, Heston WD, Gaudin PB. Comparison of anti-prostate-specific membrane antigen antibodies and other immunomarkers in metastatic prostate carcinoma. Urology. 2001; 57(6):1179-1183. [PubMed: 11377343]

5. Bander NH, Milowsky MI, Nanus DM, Kostakoglu L, Vallabhajosula S, Goldsmith SJ. Phase I trial of 177lutetium-labeled J591, a monoclonal antibody to prostate-specific membrane antigen, in patients with androgen-independent prostate cancer. J. Clin. Oncol. 2005; 23(21):4591-4601. [PubMed: 15837970]

6. Perner S, Hofer MD, Kim R, Shah RB, Li H, Moller P, Hautmann RE, Gschwend JE, Kuefer R, Rubin MA. Prostate-specific membrane antigen expression as a predictor of prostate cancer progression. Hum. Pathol. 2007; 38(5):696-701. [PubMed: 17320151]

7. (a) Byun, Y.; Mease, RC.; Lupold, S.; Pomper, MG. Recent Developments of Diagnostic and Therapeutic Agents Targeting Glutamate Carboxypeptidase II (GCPII). In: Supuran, CT.; Winum, JY., editors. Drug Design of Zinc-Enzyme Inhibitors: Functional Structureal and Disease Applications. Hoboken, NJ: John Wiley \&, Sons; 2009. p. 881-910.(b) Tsukamoto T, Wozniak KM, Slusher BS. Progress in the discovery and development of glutamate carboxypeptidase II inhibitors. Drug Discovery Today. 2007; 12(17-18):767-776. [PubMed: 17826690] (c) Zhou J, Neale JH, Pomper MG, Kozikowski AP. NAAG peptidase inhibitors and their potential for diagnosis and therapy. Nat. Rev. Drug Discovery. 2005; 4(12):1015-1026. [PubMed: 16341066]

8. (a) Jackson PF, Cole DC, Slusher BS, Stetz SL, Ross LE, Donzanti BA, Trainor DA. Design, synthesis, and biological activity of a potent inhibitor of the neuropeptidase N-acetylated alphalinked acidic dipeptidase. J. Med. Chem. 1996; 39(2):619-622. [PubMed: 8558536] (b) Jackson PF, Tays KL, Maclin KM, Ko YS, Li W, Vitharana D, Tsukamoto T, Stoermer D, Lu XC, Wozniak K, Slusher BS. Design and pharmacological activity of phosphinic acid based NAALADase inhibitors. J. Med. Chem. 2001; 44(24):4170-4175. [PubMed: 11708918]

9. Maung J, Mallari JP, Girtsman TA, Wu LY, Rowley JA, Santiago NM, Brunelle AN, Berkman CE. Probing for a hydrophobic a binding register in prostate-specific membrane antigen with phenylalkylphosphonamidates. Bioorg. Med. Chem. 2004; 12(18):4969-4979. [PubMed: 15336276]

10. (a) Kozikowski AP, Nan F, Conti P, Zhang J, Ramadan E, Bzdega T, Wroblewska B, Neale JH, Pshenichkin S, Wroblewski JT. Design of remarkably simple, yet potent urea-based inhibitors of glutamate carboxypeptidase II (NAALADase). J. Med. Chem. 2001; 44(3):298-301. [PubMed: 11462970] (b) Kozikowski AP, Zhang J, Nan F, Petukhov PA, Grajkowska E, Wroblewski JT, 
Yamamoto T, Bzdega T, Wroblewska B, Neale JH. Synthesis of urea-based inhibitors as active site probes of glutamate carboxypeptidase II: efficacy as analgesic agents. J. Med. Chem. 2004; 47(7): 1729-1738. [PubMed: 15027864]

11. (a) Majer P, Jackson PF, Delahanty G, Grella BS, Ko YS, Li W, Liu Q, Maclin KM, Polakova J, Shaffer KA, Stoermer D, Vitharana D, Wang EY, Zakrzewski A, Rojas C, Slusher BS, Wozniak KM, Burak E, Limsakun T, Tsukamoto T. Synthesis and biological evaluation of thiol-based inhibitors of glutamate carboxypeptidase II: discovery of an orally active GCP II inhibitor. J. Med. Chem. 2003; 46(10):1989-1996. [PubMed: 12723961] (b) Stoermer D, Vitharana D, Hin N, Delahanty G, Duvall B, Ferraris DV, Grella BS, Hoover R, Rojas C, Shanholtz MK, Smith KP, Stathis M, Wu Y, Wozniak KM, Slusher BS, Tsukamoto T. Design, synthesis, and pharmacological evaluation of glutamate carboxypeptidase II (GCPII) inhibitors based on thioalkylbenzoic acid scaffolds. J. Med. Chem. 2012; 55(12):5922-5932. [PubMed: 22642259]

12. Stoermer D, Liu Q, Hall MR, Flanary JM, Thomas AG, Rojas C, Slusher BS, Tsukamoto T. Synthesis and biological evaluation of hydroxamate-Based inhibitors of glutamate carboxypeptidase II. Bioorg. Med. Chem. Lett. 2003; 13(13):2097-2100. [PubMed: 12798312]

13. (a) Foss CA, Mease RC, Cho SY, Kim HJ, Pomper MG. GCPII imaging and cancer. Curr. Med. Chem. 2012; 19(9):1346-1359. [PubMed: 22304713] (b) Mease RC, Foss CA, Pomper MG. PET imaging in prostate cancer: focus on prostate-specific membrane antigen. Curr. Top. Med. Chem. 2013; 13(8):951-962. [PubMed: 23590171] (c) Vargas HA, Grimm J, Donati OF, Sala E, Hricak $\mathrm{H}$. Molecular imaging of prostate cancer: translating molecular biology approaches into the clinical realm. European radiology. 2015; 25:1294-1302. [PubMed: 25693661] (d) Chen Y, Foss CA, Byun Y, Nimmagadda S, Pullambhatla M, Fox JJ, Castanares M, Lupold SE, Babich JW, Mease RC, Pomper MG. Radiohalogenated Prostate-Specific Membrane Antigen (PSMA)-Based Ureas as Imaging Agents for Prostate Cancer. J. Med. Chem. 2008; 51(24):7933-7943. [PubMed: 19053825] (e) Barinka C, Byun Y, Dusich CL, Banerjee SR, Chen Y, Castanares M, Kozikowski AP, Mease RC, Pomper MG, Lubkowski J. Interactions between human glutamate carboxypeptidase II and urea-based inhibitors: structural characterization. J. Med. Chem. 2008; 51(24):7737-7743. [PubMed: 19053759]

14. (a) Banerjee SR, Foss CA, Castanares M, Mease RC, Byun Y, Fox JJ, Hilton J, Lupold SE, Kozikowski AP, Pomper MG. Synthesis and evaluation of technetium-99m- and rhenium-labeled inhibitors of the prostate-specific membrane antigen (PSMA). J. Med. Chem. 2008; 51(15):45044517. [PubMed: 18637669] (b) Banerjee SR, Foss CA, Pullambhatla M, Wang Y, Srinivasan S, Hobbs R, Baidoo KE, Brechbiel MW, Nimmagadda S, Mease RC, Sgouros G, Pomper MG. Preclinical Evaluation of 86Y-Labeled Inhibitors of Prostate-Specific Membrane Antigen for Dosimetry Estimates. J. Nucl. Med. 2015; 56:628-634. [PubMed: 25722448] (c) Banerjee SR, Pullambhatla M, Byun Y, Nimmagadda S, Green G, Fox JJ, Horti A, Mease RC, Pomper MG. 68Ga-labeled inhibitors of prostate-specific membrane antigen (PSMA) for imaging prostate cancer. J. Med. Chem. 2010; 53(14):5333-5341. [PubMed: 20568777] (d) Banerjee SR, Pullambhatla M, Foss CA, Nimmagadda S, Ferdani R, Anderson CJ, Mease RC, Pomper MG. ${ }^{64} \mathrm{Cu}$-labeled inhibitors of prostate-specific membrane antigen for PET imaging of prostate cancer. J. Med. Chem. 2014; 57(6):2657-2669. [PubMed: 24533799] (e) Chen Y, Pullambhatla M, Byun Y, Foss CA, Nimmagadda S, Senthamizhchelvan S, Sgouros G, Mease RC, Pomper MG. 2-(3-\{1Carboxy-5-[(6-[18F]fluoro-pyridine-3-carbonyl)-amino]-pentyl \}-urei do)-pentanedioic acid, [18F]DCFPyL, a PSMA-based PET Imaging Agent for Prostate Cancer. Clin. Cancer Res. 2011; 17:7645-7653. [PubMed: 22042970] (f) Eder M, Schafer M, Bauder-Wust U, Hull WE, Wangler C, Mier W, Haberkorn U, Eisenhut M. 68Ga-complex lipophilicity and the targeting property of a urea-based PSMA inhibitor for PET imaging. Bioconjugate Chem. 2012; 23(4):688-697.(g) Foss CA, Mease RC, Fan H, Wang Y, Ravert HT, Dannals RF, Olszewski RT, Heston WD, Kozikowski AP, Pomper MG. Radiolabeled small-molecule ligands for prostate-specific membrane antigen: in vivo imaging in experimental models of prostate cancer. Clin. Cancer Res. 2005; 11(11):40224028. [PubMed: 15930336] (h) Hillier SM, Maresca KP, Lu G, Merkin RD, Marquis JC, Zimmerman CN, Eckelman WC, Joyal JL, Babich JW. 99mTc-labeled small-molecule inhibitors of prostate-specific membrane antigen for molecular imaging of prostate cancer. J. Nucl. Med. 2013; 54(8):1369-1376. [PubMed: 23733925] (i) Kularatne SA, Zhou Z, Yang J, Post CB, Low PS. Design, synthesis, and preclinical evaluation of prostate-specific membrane antigen targeted (99m)Tc-radioimaging agents. Mol. Pharmaceutics. 2009; 6(3):790-800.(j) Lapi SE, Wahnishe H, Pham D, Wu LY, Nedrow-Byers JR, Liu T, Vejdani K, VanBrocklin HF, Berkman CE, Jones EF. 
Assessment of an 18F-labeled phosphoramidate peptidomimetic as a new prostate-specific membrane antigen-targeted imaging agent for prostate cancer. J. Nucl. Med. 2009; 50(12):20422048. [PubMed: 19910433] (k) Maresca KP, Hillier SM, Femia FJ, Keith D, Barone C, Joyal JL, Zimmerman CN, Kozikowski AP, Barrett JA, Eckelman WC, Babich JW. A series of halogenated heterodimeric inhibitors of prostate specific membrane antigen (PSMA) as radiolabeled probes for targeting prostate cancer. J. Med. Chem. 2009; 52(2):347-357. [PubMed: 19111054] (1) Mease RC, Dusich CL, Foss CA, Ravert HT, Dannals RF, Seidel J, Prideaux A, Fox JJ, Sgouros G, Kozikowski AP, Pomper MG. N-[N-[(S)-1,3-Dicarboxypropyl]carbamoyl]-4-[18F]-fluorobenzylL-cysteine, [18F]DCFBC: a new imaging probe for prostate cancer. Clin. Cancer Res. 2008; 14(10):3036-3043. [PubMed: 18483369] (m) Ray Banerjee S, Pullambhatla M, Foss CA, Falk A, Byun Y, Nimmagadda S, Mease RC, Pomper MG. Effect of chelators on the pharmacokinetics of $(99 \mathrm{~m}) \mathrm{Tc}-\mathrm{labeled}$ imaging agents for the prostate-specific membrane antigen (PSMA). J. Med. Chem. 2013; 56(15):6108-6121. [PubMed: 23799782] (n) Weineisen M, Simecek J, Schottelius M, Schwaiger M, Wester H-J. Synthesis and preclinical evaluation of DOTAGA-conjugated PSMA ligands for functional imaging and endoradiotherapy of prostate cancer. EJNMMI Res. 2014; 4

15. (a) Afshar-Oromieh A, Avtzi E, Giesel FL, Holland-Letz T, Linhart HG, Eder M, Eisenhut M, Boxler S, Hadaschik BA, Kratochwil C, Weichert W, Kopka K, Debus J, Haberkorn U. The diagnostic value of PET/CT imaging with the (68)Ga-labelled PSMA ligand HBED-CC in the diagnosis of recurrent prostate cancer. Eur. J. Nucl. Med. Mol. Imaging. 2015; 42(2):197-209. [PubMed: 25411132] (b) Afshar-Oromieh A, Haberkorn U, Eder M, Eisenhut M, Zechmann CM. [68Ga]Gallium-labelled PSMA ligand as superior PET tracer for the diagnosis of prostate cancer: comparison with 18F-FECH. Eur. J. Nucl. Med. Mol. Imaging. 2012; 39(6):1085-1086. [PubMed: 22310854] (c) Afshar-Oromieh A, Malcher A, Eder M, Eisenhut M, Linhart HG, Hadaschik BA, Holland-Letz T, Giesel FL, Kratochwil C, Haufe S, Haberkorn U, Zechmann CM. PET imaging with a [68Ga]gallium-labelled PSMA ligand for the diagnosis of prostate cancer: biodistribution in humans and first evaluation of tumour lesions. Eur. J. Nucl. Med. Mol. Imaging. 2013; 40(4):486495. [PubMed: 23179945] (d) Afshar-Oromieh A, Zechmann CM, Malcher A, Eder M, Eisenhut M, Linhart HG, Holland-Letz T, Hadaschik BA, Giesel FL, Debus J, Haberkorn U. Comparison of PET imaging with a (68)Ga-labelled PSMA ligand and (18)F-choline-based PET/CT for the diagnosis of recurrent prostate cancer. Eur. J. Nucl. Med. Mol. Imaging. 2014; 41(1):11-20. [PubMed: 24072344] (e) Barrett JA, Coleman RE, Goldsmith SJ, Vallabhajosula S, Petry NA, Cho S, Armor T, Stubbs JB, Maresca KP, Stabin MG, Joyal JL, Eckelman WC, Babich JW. First-inMan Evaluation of 2 High-Affinity PSMA-Avid Small Molecules for Imaging Prostate Cancer. J. Nucl. Med. 2013; 54(3):380-387. [PubMed: 23303962] (f) Cho SY, Gage KL, Mease RC, Senthamizhchelvan S, Holt DP, Jeffrey-Kwanisai A, Endres CJ, Dannals RF, Sgouros G, Lodge M, Eisenberger MA, Rodriguez R, Carducci MA, Rojas C, Slusher BS, Kozikowski AP, Pomper MG. Biodistribution, tumor detection, and radiation dosimetry of 18F-DCFBC, a low-molecular-weight inhibitor of prostate-specific membrane antigen, in patients with metastatic prostate cancer. J. Nucl. Med. 2012; 53(12):1883-1891. [PubMed: 23203246] (g) Rowe S, Gage KL, Macura K, Guner G, Faraj SF, Munari E, Rodriquez R, Han M, Blackford A, Netto G, Lodge M, Mease RC, Pomper MG, Cho SY, et al. 18F-DCFBC PET/CT for PSMA-Based Detection and Characterization of Primary Prostate Cancer. J. Nucl. Med. 2015; 56:1003. [PubMed: 26069305] (h) Szabo Z, Mena E, Rowe SP, Plyku D, Nidal R, Eisenberger MA, Antonarakis ES, Fan H, Dannals RF, Chen Y, Mease RC, Vranesic M, Bhatnagar A, Sgouros G, Cho SY, Pomper MG. Initial Evaluation of $\left[{ }^{18}\right.$ F $]$ DCFPyL for Prostate-Specific Membrane Antigen (PSMA)-Targeted PET Imaging of Prostate Cancer. Mol Imaging Biol. 2015; 17:565. [PubMed: 25896814] (i) Vallabhajosula S, Nikolopoulou A, Babich JW, Osborne JR, Tagawa ST, Lipai I, Solnes L, Maresca KP, Armor T, Joyal JL, Crummet R, Stubbs JB, Goldsmith SJ. 99mTc-labeled smallmolecule inhibitors of prostate-specific membrane antigen: pharmacokinetics and biodistribution studies in healthy subjects and patients with metastatic prostate cancer. J. Nucl. Med. 2014; 55(11):1791-1798. [PubMed: 25342385]

16. Wang EK, Kolb HC, Szardenings AK, Liu C, Walsh JC, Chen G, Sinha A, Kasi D, Yu C, Gangadharmath UB, Zhang W, Zhao T, Mocharla VP. PSMA Imaging Agents. 2013

17. Chen Y, Pullambhatla M, Banerjee SR, Byun Y, Stathis M, Rojas C, Slusher BS, Mease RC, Pomper MG. Synthesis and biological evaluation of low molecular weight fluorescent imaging agents for the prostate-specific membrane antigen. Bioconjugate Chem. 2012; 23(12):2377-2385. 
18. Kabalka GW, Mereddy AR. A facile no-carrier-added radioiodination procedure suitable for radiolabeling kits. Nucl. Med. Biol. 2004; 31(7):935-938. [PubMed: 15464395]

19. Zalutsky MR, Narula AS. A method for the radio-halogenation of proteins resulting in decreased thyroid uptake of radioiodine. Int. J. Rad Appl. Instrum Part A. 1987; 38:1051-1055.

20. Al-Darwich MJ, Plenevaux A, Lemaire C, Fiore GD, Christiaens L, Comar D, Luxen A. Enantioselective synthesis of no-carrieradded (S)-4-chloro-2- $\left[{ }^{18} \mathrm{~F}\right]$ fluorophenylalanine and (S)-amethyl)-4-chloro-2-[ ${ }^{18}$ F]fluorophenylalanine. J. Fluorine Chem. 1996; 80:117-124.

21. Glaser M, Arstad E, Luthra SK, Robins EG. Two-step radiosynthesis of [18F]N-succinimidyl-4fluorobenzoate ([ ${ }^{18}$ F]SFB. J. Labelled Compd. Radiopharm. 2009; 52:327-330.

22. (a) Zhong C, Zhao X, Sarva J, Kozikowski A, Neale JH, Lyeth BG. NAAG peptidase inhibitor reduces acute neuronal degeneration and astrocyte damage following lateral fluid percussion TBI in rats. J. Neurotrauma. 2005; 22(2):266-276. [PubMed: 15716632] (b) Wang H, Byun Y, Barinka C, Pullambhatla M, Bhang HE, Fox JJ, Lubkowski J, Mease RC, Pomper MG. Bioisosterism of urea-based GCPII inhibitors: Synthesis and structure-activity relationship studies. Bioorg. Med. Chem. Lett. 2010; 20(1):392-397. [PubMed: 19897367]

23. (a) Silver DA, Pellicer I, Fair WR, Heston WD, Cordon-Cardo C. Prostate-specific membrane antigen expression in normal and malignant human tissues. Clin. Cancer Res. 1997; 3(1):81-85. [PubMed: 9815541] (b) Slusher BS, Tsai G, Yoo G, Coyle JT. Immunocytochemical localization of the N-acetyl-aspartyl-glutamate (NAAG) hydrolyzing enzyme $\mathrm{N}$-acetylated alpha-linked acidic dipeptidase (NAALADase). J. Comp. Neurol. 1992; 315(2):217-229. [PubMed: 1545010]

24. Reske SN, Winter G, Baur B, Machulla HJ, Kull T. Comment on Afshar-Oromieh et al.: PET imaging with a [68Ga]-gallium-labelled PSMA ligand for the diagnosis of prostate cancer: biodistribution in humans and first evaluation of tumour lesions. Eur. J. Nucl. Med. Mol. Imaging. 2013; 40(6):969-970. [PubMed: 23558687]

25. Zechmann CM, Afshar-Oromieh A, Armor T, Stubbs JB, Mier W, Hadaschik B, Joyal J, Kopka K, Debus J, Babich JW, Haberkorn U. Radiation dosimetry and first therapy results with a (124)I/ (131)I-labeled small molecule (MIP-1095) targeting PSMA for prostate cancer therapy. Eur. J. Nucl. Med. Mol. Imaging. 2014; 41(7):1280-1292. [PubMed: 24577951]

26. Afshar-Oromieh A, Haberkorn U, Hadaschik B, Habl G, Eder M, Eisenhut M, Schlemmer HP, Roethke MC. PET/MRI with a 68Ga-PSMA ligand for the detection of prostate cancer. Eur. J. Nucl. Med. Mol. Imaging. 2013; 40(10):1629-1630. [PubMed: 23817686]

27. Kratochwil C, Giesel FL, Eder M, Afshar-Oromieh A, Benesova M, Mier W, Kopka K, Haberkorn U. $\left[{ }^{177} \mathrm{Lu}\right]$ Lutetium-labelled PSMA ligand-induced remission in a patient with metastatic prostate cancer. Eur. J. Nucl. Med. Mol. Imaging. 2015; 42:987. [PubMed: 25573634]

28. Makarasen A, Nishikawa T, Isobe M. Synthesis of four lysine-linked cereulide analogues showing ionophoric activity towards potassium cations as lead compounds for emetic toxin detection by immunoassays. Synthesis. 2009; 2009:2184-2004.

29. Litosh VA, Hersh MN, Stupi BP, Wu W, Metzker ML. Nucleotides and nucleosides and methods for their use in DNA sequencing. Patent WO2009152353 A2. 2009

30. Winkler JW, Uddin J, Serhan CN, Petasis NA. Stereocontrolled total synthesis of the potent antiinflammatory and pro-resolving lipid mediator resolvin D3 and its aspirin-triggered 17R-epimer. Org. Lett. 2013; 15(7):1424-1427. [PubMed: 23510485]

31. Cheng Y, Prusoff WH. Relationship between the inhibition constate (K1) and the concentration of inhibitor which causes 50 percent inhibition (I50) of an enzymatic reaction. Biochem. Pharmacol. 1973; 22:3099-3108. [PubMed: 4202581]

32. Chang SS, Reuter VE, Heston WD, Bander NH, Grauer LS, Gaudin PB. Five different antiprostate-specific membrane antigen (PSMA) antibodies confirm PSMA expression in tumorassociated neovasculature. Cancer Res. 1999; 59(13):3192-3198. [PubMed: 10397265] 
<smiles></smiles><smiles>CC(C(=O)OO)C(=O)C(=O)C(=O)OO</smiles>
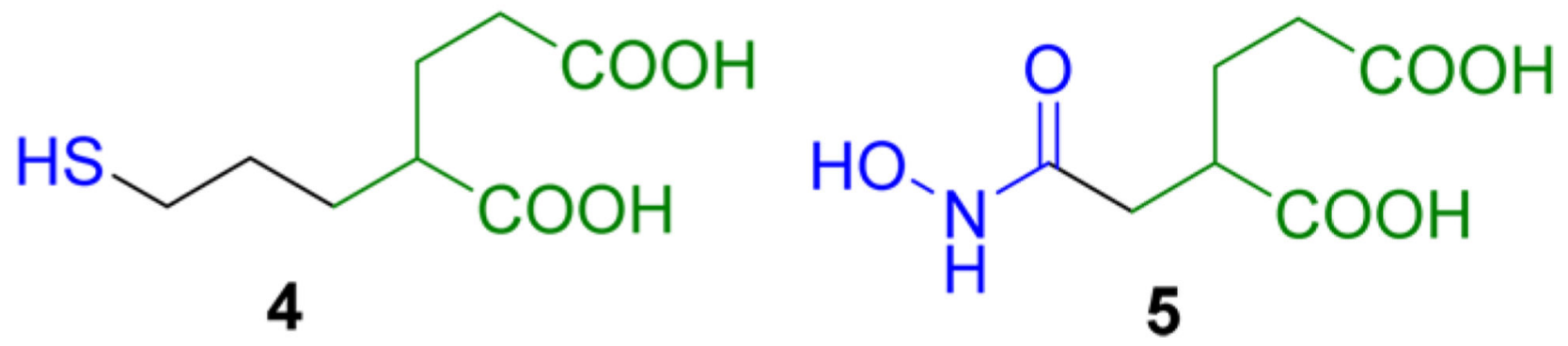

Figure 1.

PSMA binding scaffolds.

$3 X=\mathrm{NH}, \mathrm{Y}=\mathrm{NH}$

$6 \mathrm{X}=\mathrm{O}, \mathrm{Y}=\mathrm{NH}$

$7 \mathrm{X}=\mathrm{NH}, \mathrm{Y}=\mathrm{O}$ 
<smiles>[Y][Y](=O)[Y]#N</smiles><smiles>[X]c1ccc(C(=O)NCCCC[C@H](NC(=O)N[C@@H](CCC(=O)O)C(=O)O)C(=O)O)cc1</smiles>

$9 X=\mathrm{F}$

$10 X=1$

Figure 2.

Ureas and carbamates. 

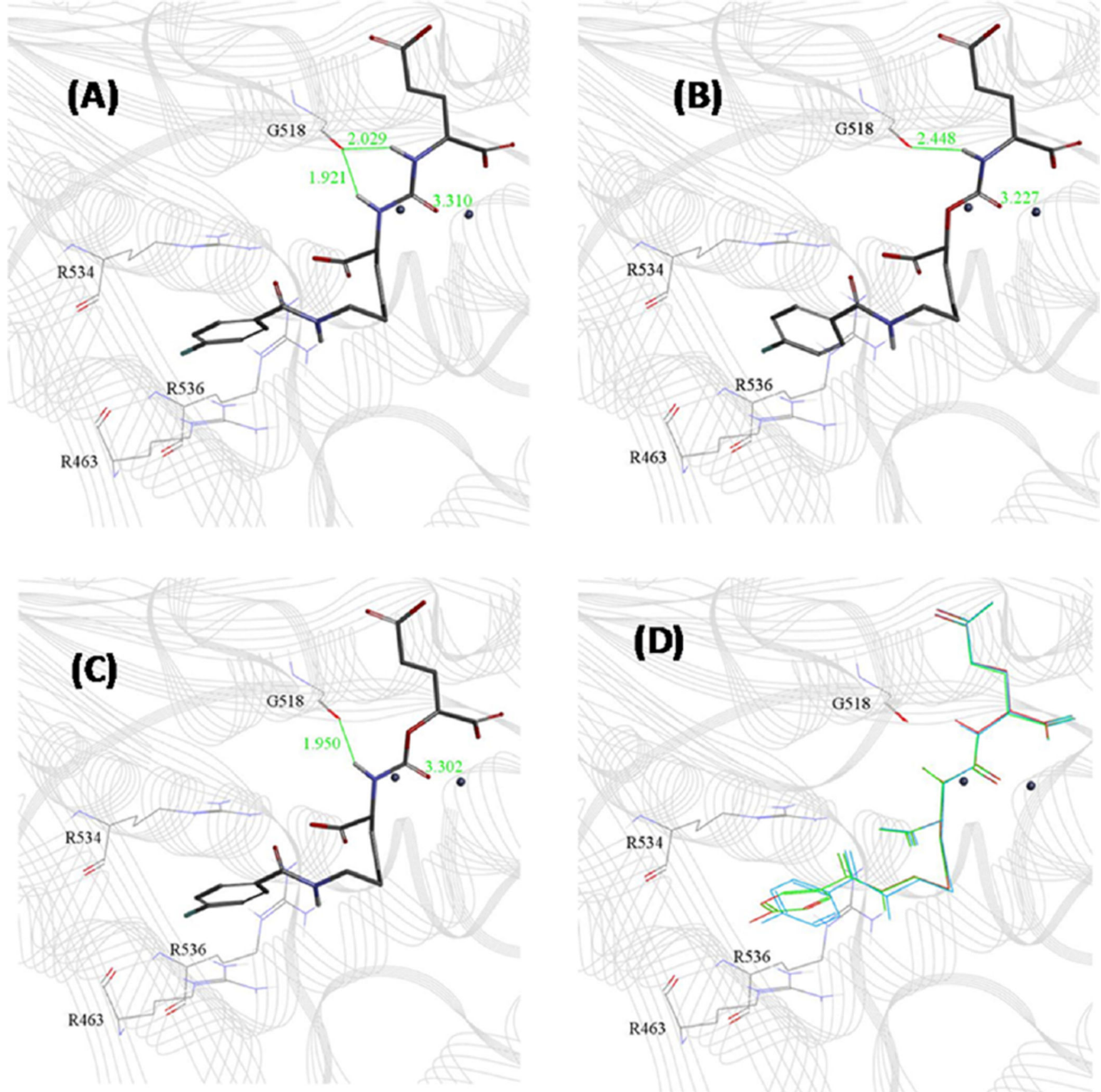

Figure 3.

Docking of 9, 12, and 13 to PSMA (PDB code 3D7H): (A) urea 9; (B) Lys-NPA carbamate 12; (C) Lys-OPA carbamate 13; (D) overlay of 9 (red), 12 (blue), and 13 (green). 

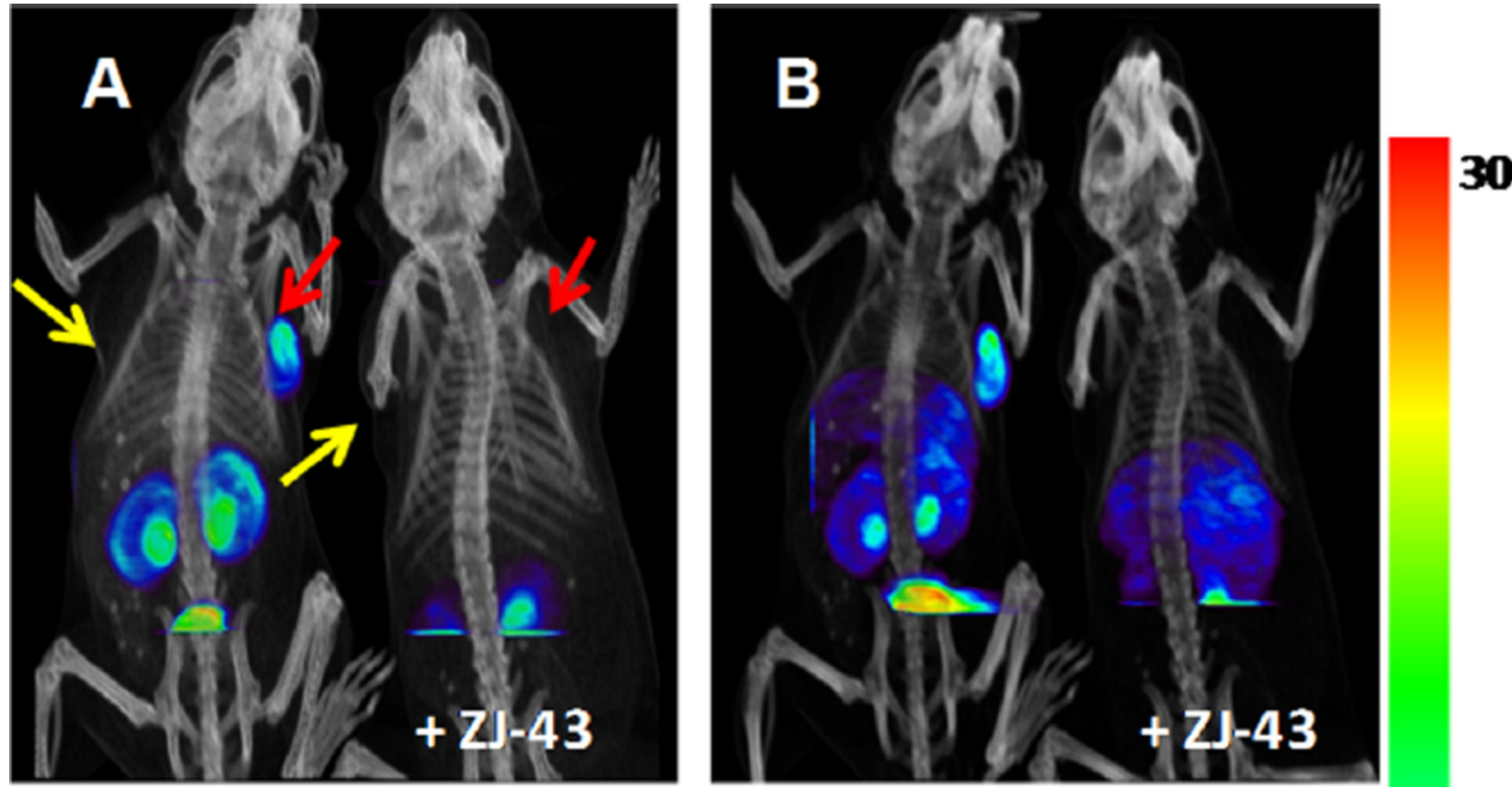

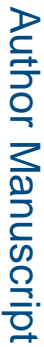
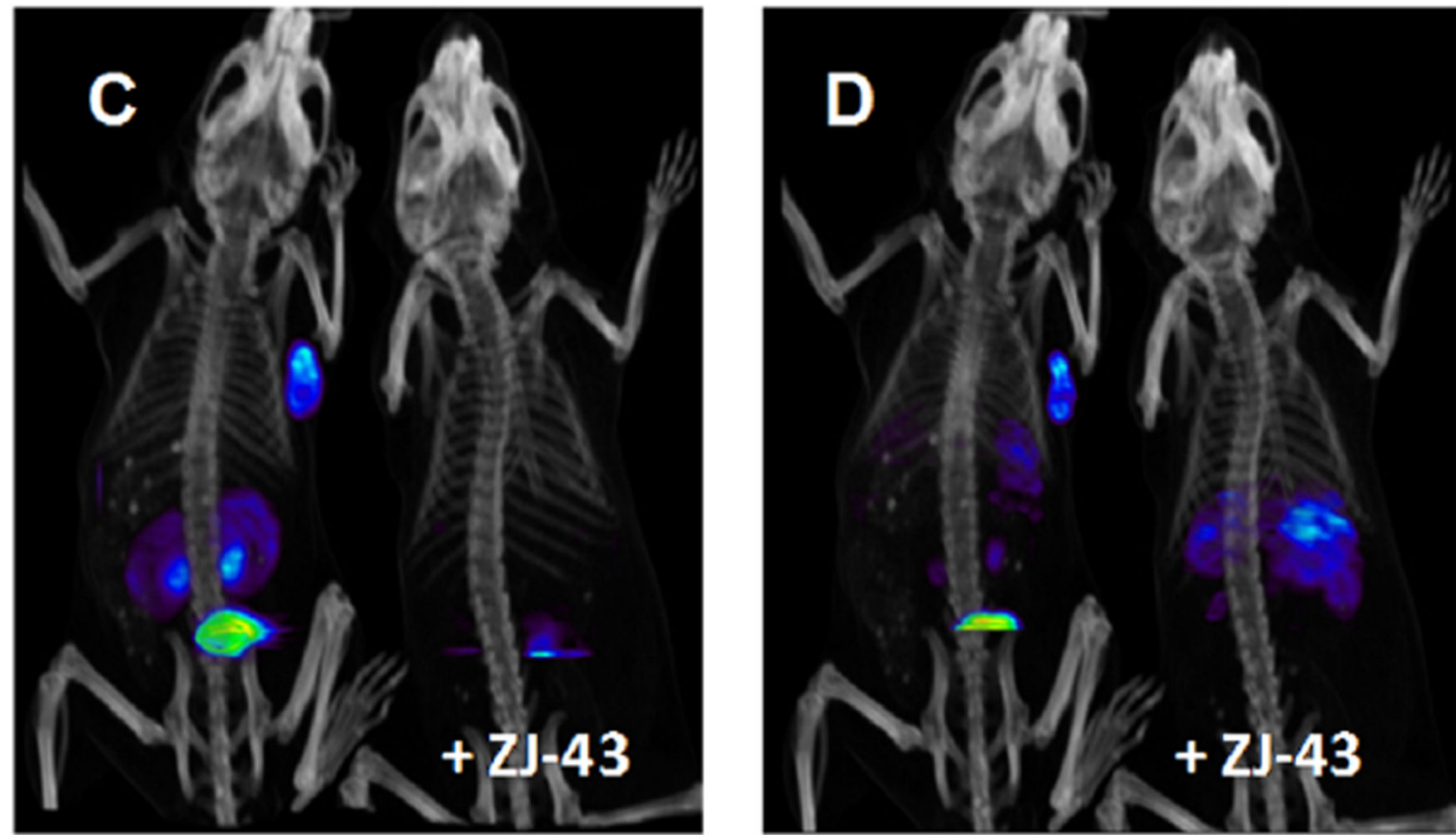

0 \%ID/cc

Figure 4.

Whole body PET/CT images of tumor bearing mice [PSMA + PC3 PIP (red arrows) and PSMA- PC3 flu (yellow arrows)] in tumor bearing mice. The left mouse in each panel was injected with $740 \mathrm{kBq}(200 \mu \mathrm{Ci})\left[{ }^{18} \mathrm{~F}\right] \mathbf{1 2}$. The right mouse was co-injected with $740 \mathrm{kBq}$ $(200 \mu \mathrm{Ci})\left[{ }^{18} \mathrm{~F}\right] \mathbf{1 2}$ and $25 \mathrm{mg} / \mathrm{kg}$ of the competing PSMA inhibitor ZJ-43. (A) 5-15 min image; (B) 15-25 min image; (C) 25-35 min image; (D) 50-60 min image. 


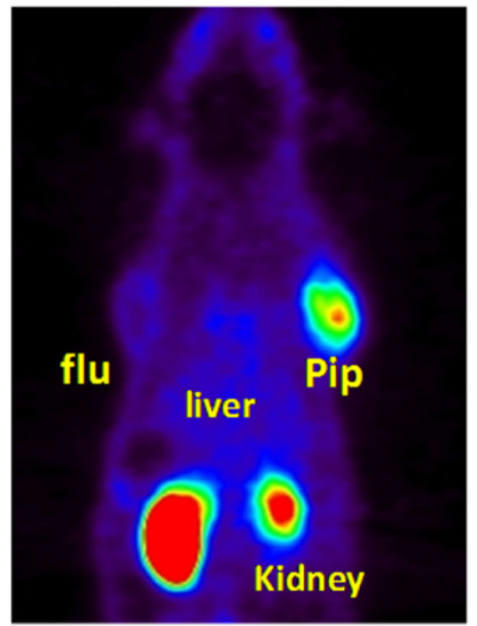

$10 \mathrm{~min}$

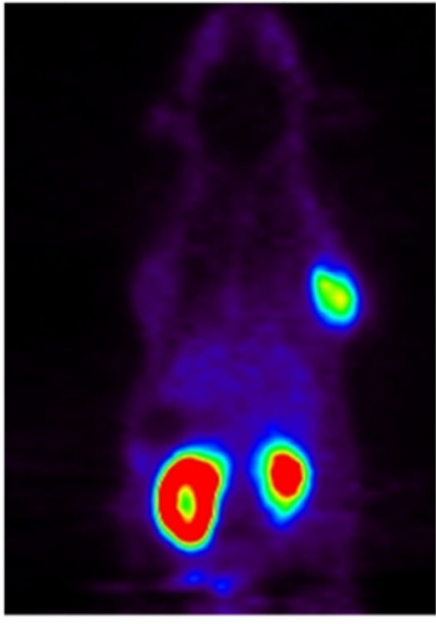

30 min

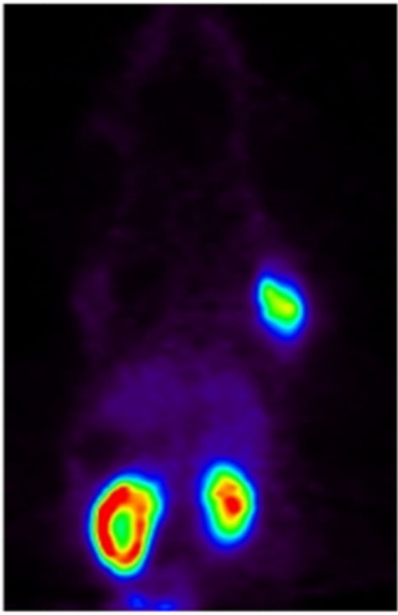

$60 \mathrm{~min}$

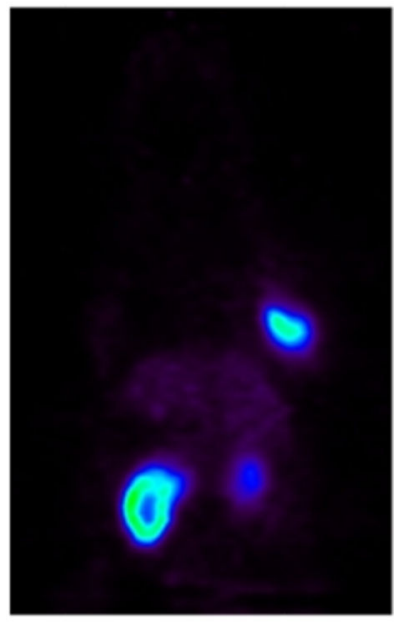

$120 \mathrm{~min}$

\section{$\begin{array}{lll}0 & \text { \%D/cc } & 25\end{array}$}

Figure 5.

Sequential small animal PET (coronal view) images of $\left[{ }^{18} \mathrm{~F}\right] \mathbf{1 3}$ in a male SCID mouse containing PSMA+ PC3 PIP and PSMA- PC3 flu tumor xenografts. 


\section{$\left[{ }^{18} \mathrm{~F}\right] 23$}

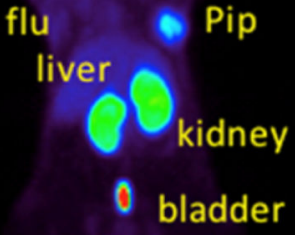

10-30 min

$\left[{ }^{18} \mathrm{~F}\right] 24$

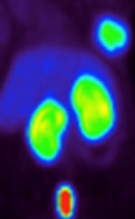

0

30-50 min

$60-80 \mathrm{~min}$

$2 \mathrm{~h}$

$4 \mathrm{~h}$
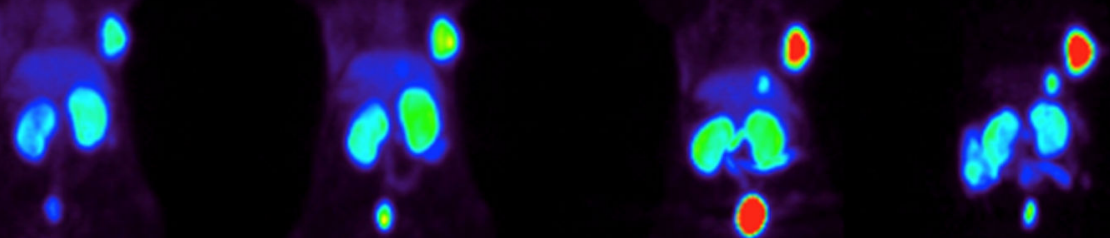

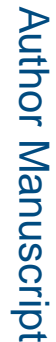

0

SUV

Figure 6.

Sequential whole body micro-PET images of $\left[{ }^{18} \mathrm{~F}\right] \mathbf{2 3}$ (top row) and $\left[{ }^{18} \mathrm{~F}\right] \mathbf{2 4}$ (bottom row) in male SCID mice bearing PSMA+ PC3 PIP and PSMA- PC3 flu tumor xenografts. 


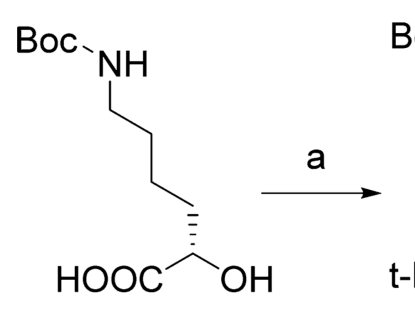

14<smiles>CCCCOC(=O)C(O)CCCCNC(=O)OCc1ccccc1</smiles><smiles>CCCCOC(=O)[C@H](CCCCNC(=O)OCc1ccccc1)OC(=O)n1ccnc1</smiles>

Boc、<smiles>[AlH2]</smiles>

16

17

15

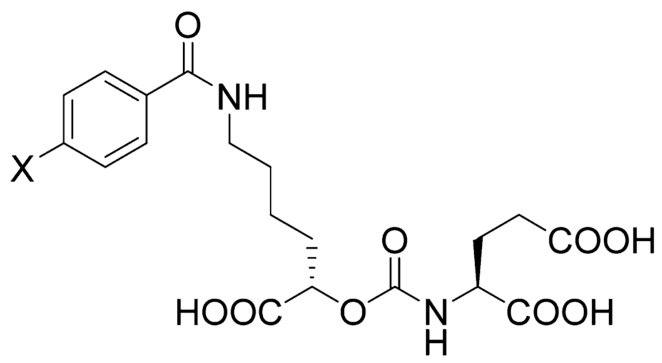

18

$$
\begin{aligned}
& 12 X=F \text { or }{ }^{18} \mathrm{~F} \\
& 31 \mathrm{X}=\mathrm{I} \text { or }{ }^{125} \mathrm{I}
\end{aligned}
$$

Scheme $1^{a}$

${ }^{a}$ Reagents and conditions: (a) tert-butyl-1,3-diisopropylisourea, $\mathrm{CH}_{2} \mathrm{Cl}_{2}, \mathrm{rt}, 3 \mathrm{~d}$; (b) carbonyldiimidazole, $\mathrm{CH}_{2} \mathrm{Cl}_{2}$, rt, $1 \mathrm{~h}$; (c) L-glutamic acid di-tert-butyl ester, neat, $45^{\circ} \mathrm{C}, 2 \mathrm{~d}$; (d) TFA/ $\mathrm{CH}_{2} \mathrm{Cl}_{2}$ (1/1), rt, $2 \mathrm{~h}$; (e) $\mathrm{N}$-succinimidyl-4-fluorobenzoate or $\mathrm{N}$-succinimidyl-4$\left[{ }^{18} \mathrm{~F}\right]$ fluorobenzoate, $\mathrm{Et}_{3} \mathrm{~N}$, DMSO, rt, $2 \mathrm{~h}$; (f) $\mathrm{N}$-succinimidyl-4-iodobenzoate or $\mathrm{N}$ succinimidyl-4-[ $\left.{ }^{125} 1\right]$ iodobenzoate, diisopropylethylamine, DMSO rt, $1 \mathrm{~h}$. 
<smiles>COC(=O)c1ccccc1</smiles>

19

20

\section{1}

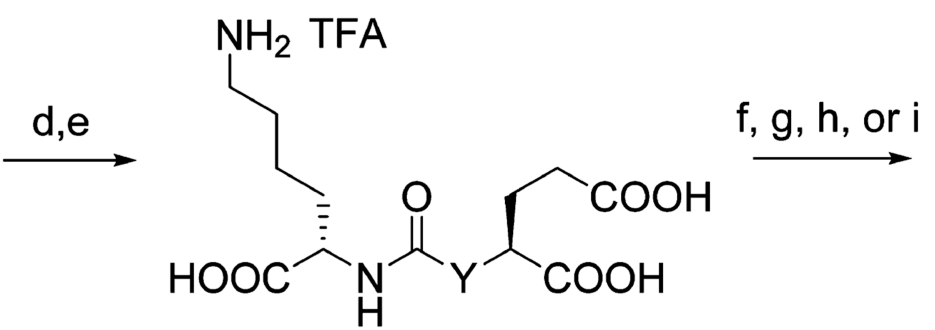

$$
\begin{aligned}
& 22 Y=0 \\
& 25 Y=N H
\end{aligned}
$$<smiles>[Y]C(=O)N[C@@H](CCCCNC(=O)c1ccc([Z])cc1[Y])C(=O)O</smiles><smiles>[Y]C(=O)N[C@@H](CCCCNC(=O)c1ccc([Z])cc1[Y])C(=O)O</smiles>

$13 \mathrm{Y}=\mathrm{O}, \mathrm{Z}=\mathrm{F}$ or ${ }^{18} \mathrm{~F}, \mathrm{~W}=\mathrm{H}$

$32 Y=O, Z=I$ or ${ }^{125} \mathrm{I}, \mathrm{W}=\mathrm{H}$

$23 \mathrm{Y}=\mathrm{O}, \mathrm{Z}=\mathrm{Br}, \mathrm{W}=\mathrm{F}$

$24 \mathrm{Y}=\mathrm{O}, \mathrm{Z}=\mathrm{I}, \mathrm{W}=\mathrm{F}$

$26 \mathrm{Y}=\mathrm{NH}, \mathrm{Z}=\mathrm{Br}, \mathrm{W}=\mathrm{F}$

$27 \mathrm{Y}=\mathrm{NH}, \mathrm{Z}=\mathrm{I}, \mathrm{W}=\mathrm{F}$

\section{Scheme $2^{a}$}

${ }^{a}$ Reagents and conditions: (a) carbonyldiimidazole, $\mathrm{CH}_{2} \mathrm{Cl}_{2}$, rt, $1 \mathrm{~h}$; (b) $\mathrm{Mel}, \mathrm{MeCN}, 55^{\circ} \mathrm{C}$,

$3 \mathrm{~h}$; (c) N-Boc-lysine-tert-butyl ester hydrochloride, $\mathrm{Et}_{3} \mathrm{~N}$, DMSO, rt, overnight; (d)

TFA/ $\mathrm{CH}_{2} \mathrm{Cl}_{2}$ (1/1), rt, $2 \mathrm{~h}$; (e) LiOH, THF/ $\mathrm{H}_{2} \mathrm{O}$ (1/1), rt, 4 h; (f) $N$-succinimidyl-4-

fluorobenzoate or $N$-succinimidyl-4-[ $\left.{ }^{18} \mathrm{~F}\right]$ fluorobenzoate, $\mathrm{Et}_{3} \mathrm{~N}$, DMSO, rt, $2 \mathrm{~h}$; (g) $\mathrm{N}$ succinimidyl-4-[ ${ }^{125}$ I]iodobenzoate or $N$-succinimidyl-4-iodobenzoate,

diisopropylethylamine, DMSO, rt, $1 \mathrm{~h}$; (h) $\mathrm{N}$-succinimidyl 4-bromo-2-fluorobenzoate, $\mathrm{Et}_{3} \mathrm{~N}$, DMSO, rt, 2 h; (i) $N$-succinimidyl 4-iodo-2-fluorobenzoate, Et 3 N, DMSO, rt, 2 h. 
<smiles>[X][X]C(=O)C(=O)N1C(=O)CCC1=O</smiles>

Scheme $3^{a}$

${ }^{a}$ Reagents and conditions: (a) $\mathrm{K}_{222}, \mathrm{KHCO}_{3}$, DMSO, $120{ }^{\circ} \mathrm{C}, 20 \mathrm{~min}$; (b) $\mathrm{N}$ -

hydroxysuccinimide, $\mathrm{Phl}(\mathrm{OAc})_{2}, \mathrm{MeCN}, \mathrm{rt}, 15 \mathrm{~min}$; (c) 22, diisopropylethylamine, DMSO, $50{ }^{\circ} \mathrm{C}, 15 \mathrm{~min}$. 


\section{Table 1}

\begin{tabular}{|c|c|c|c|c|}
\hline \multirow{7}{*}{ 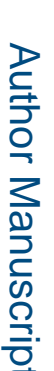 } & \multicolumn{4}{|c|}{ PSMA Inhibitory Activity } \\
\hline & compd & $K_{\mathrm{i}}(\mathrm{nM})$ & compd & $K_{\mathrm{i}}(\mathrm{nM})$ \\
\hline & $\mathbf{8}^{10 \mathrm{a}}$ & $>1000$ & 23 & 0.11 \\
\hline & $9^{13 \mathrm{~d}}$ & 0.25 & 24 & 0.21 \\
\hline & $10^{13 d}$ & 0.01 & 26 & 0.04 \\
\hline & $11^{16}$ & $>10000$ & 27 & 0.02 \\
\hline & 12 & 42 & 31 & 0.9 \\
\hline & 13 & 9.2 & 32 & 0.04 \\
\hline
\end{tabular}

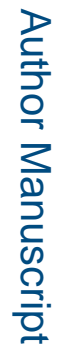

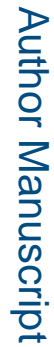

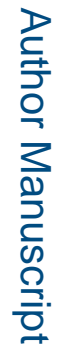

JMed Chem. Author manuscript; available in PMC 2016 May 04. 


\section{Table 2}

Biodistribution of $\left[{ }^{18} \mathrm{~F}\right] 12$ in Tumor Bearing Mice ${ }^{a}$

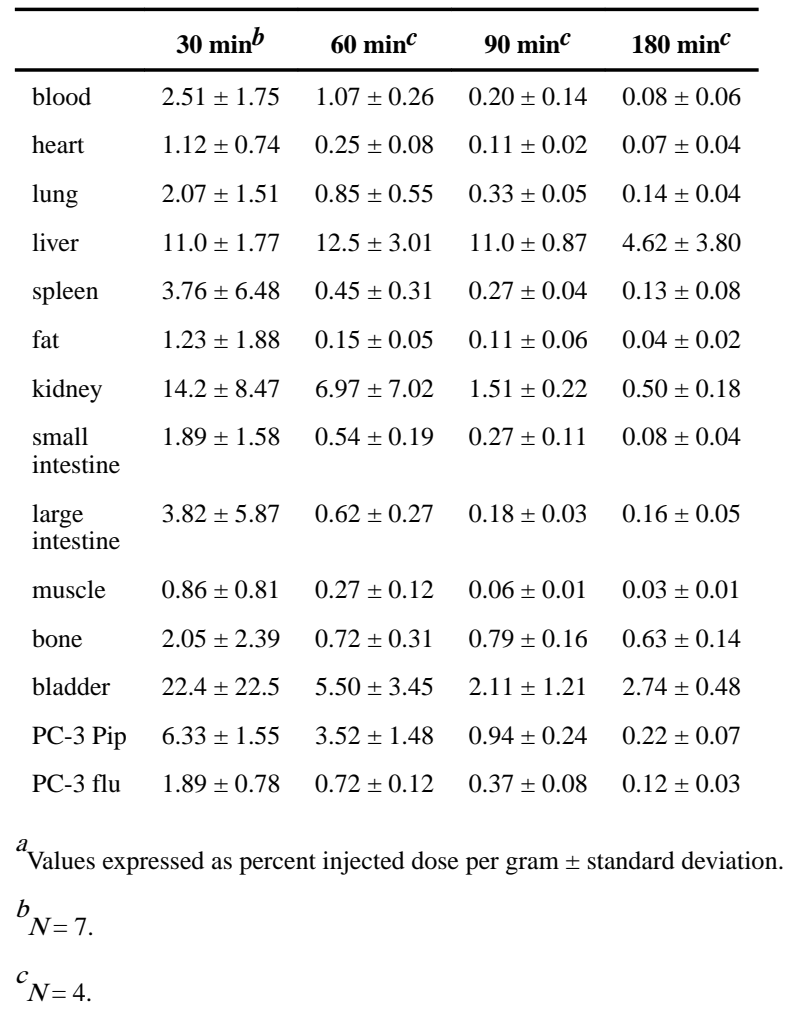


Table 3

\begin{tabular}{|c|c|c|c|c|c|}
\hline \multirow{7}{*}{ 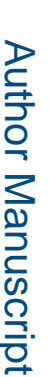 } & \multicolumn{5}{|c|}{ Biodistribution of $\left[{ }^{18} \mathrm{~F}\right] 23$ in Tumor Bearing Mice ${ }^{a}$} \\
\hline & & $0.5 \mathrm{~h}$ & $1 \mathrm{~h}$ & $2 \mathrm{~h}$ & $4 h$ \\
\hline & Blood & $4.69 \pm 0.33$ & $1.60 \pm 1.17$ & $0.61 \pm 0.41$ & $0.31 \pm 0.09$ \\
\hline & Heart & $1.72 \pm 0.31$ & $0.79 \pm 0.14$ & $0.35 \pm 0.07$ & $0.14 \pm 0.08$ \\
\hline & lung & $4.59 \pm 0.78$ & $2.62 \pm 0.30$ & $1.21 \pm 0.14$ & $0.58 \pm 0.32$ \\
\hline & liver & $15.5 \pm 1.57$ & $11.9 \pm 2.95$ & $6.42 \pm 1.06$ & $2.95 \pm 1.97$ \\
\hline & stomach & $1.08 \pm 0.07$ & $0.67 \pm 0.18$ & $0.67 \pm 0.72$ & $0.12 \pm 0.07$ \\
\hline & pancreas & $1.45 \pm 0.15$ & $0.80 \pm 0.21$ & $0.37 \pm 0.07$ & $0.13 \pm 0.03$ \\
\hline & spleen & $19.9 \pm 7.80$ & $9.62 \pm 5.53$ & $5.69 \pm 2.14$ & $1.68 \pm 1.03$ \\
\hline & fat & $1.02 \pm 0.12$ & $0.79 \pm 0.74$ & $0.88 \pm 0.80$ & $0.53 \pm 0.27$ \\
\hline & kidney & $135 \pm 23.0$ & $139 \pm 23.7$ & $127 \pm 37.1$ & $54.5 \pm 22.2$ \\
\hline & small intestine & $1.51 \pm 0.71$ & $1.67 \pm 0.70$ & $0.52 \pm 0.17$ & $0.23 \pm 0.05$ \\
\hline & large intestine & $1.35 \pm 0.35$ & $0.71 \pm 0.35$ & $0.51 \pm 0.30$ & $0.81 \pm 0.15$ \\
\hline & muscle & $0.68 \pm 0.07$ & $0.31 \pm 0.05$ & $0.14 \pm 0.02$ & $0.09 \pm 0.07$ \\
\hline & bone & $0.89 \pm 0.14$ & $0.48 \pm 0.17$ & $0.20 \pm 0.03$ & $0.09 \pm 0.05$ \\
\hline & bladder & $2.84 \pm 0.65$ & $5.11 \pm 3.57$ & $4.22 \pm 1.58$ & $3.30 \pm 3.03$ \\
\hline & PC-3 PIP & $63.4 \pm 7.16$ & $64.5 \pm 7.71$ & $90.0 \pm 13.6$ & $74.4 \pm 9.19$ \\
\hline & PC-3 flu & $2.21 \pm 0.19$ & $0.93 \pm 0.19$ & $0.39 \pm 0.01$ & $0.16 \pm 0.03$ \\
\hline & PIP:flu & 28.7 & 69.4 & 233.4 & 461.5 \\
\hline & PIP:kidney & 0.5 & 0.5 & 0.7 & 1.4 \\
\hline & PIP:muscle & 93.3 & 208.1 & 643.1 & 791.9 \\
\hline & PIP:blood & 13.5 & 40.3 & 147.6 & 240.1 \\
\hline
\end{tabular}

${ }^{a}$ Biodistribution values are expressed as percent injected dose per gram \pm standard deviation. 


\section{Table 4}

\begin{tabular}{|c|c|c|c|c|c|}
\hline \multirow{7}{*}{ 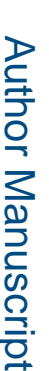 } & \multicolumn{5}{|c|}{ Biodistribution of $\left[{ }^{18} \mathrm{~F}\right] 24$ in Tumor Bearing Mice ${ }^{a}$} \\
\hline & & $0.5 \mathrm{~h}$ & 1h & $2 \mathrm{~h}$ & $4 \mathrm{~h}$ \\
\hline & blood & $7.94 \pm 0.99$ & $2.90 \pm 1.98$ & $1.29 \pm 0.87$ & $0.62 \pm 0.10$ \\
\hline & Heart & $2.27 \pm 0.32$ & $1.23 \pm 0.22$ & $0.57 \pm 0.09$ & $0.24 \pm 0.04$ \\
\hline & lung & $5.13 \pm 0.29$ & $3.31 \pm 0.41$ & $1.91 \pm 0.29$ & $0.94 \pm 0.06$ \\
\hline & liver & $16.6 \pm 1.61$ & $15.5 \pm 2.00$ & $8.99 \pm 0.55$ & $2.77 \pm 0.49$ \\
\hline & stomach & $1.80 \pm 0.08$ & $0.99 \pm 0.17$ & $0.51 \pm 0.07$ & $0.19 \pm 0.03$ \\
\hline & pancreas & $1.78 \pm 0.15$ & $1.23 \pm 0.29$ & $0.92 \pm 0.40$ & $0.26 \pm 0.06$ \\
\hline & spleen & $14.6 \pm 4.41$ & $7.81 \pm 4.22$ & $6.78 \pm 1.25$ & $3.49 \pm 0.86$ \\
\hline & fat & $1.65 \pm 0.67$ & $0.88 \pm 0.68$ & $1.12 \pm 0.43$ & $0.58 \pm 0.17$ \\
\hline & kidney & $94.4 \pm 8.22$ & $122 \pm 21.9$ & $115 \pm 13.2$ & $74.4 \pm 13.8$ \\
\hline & small intestine & $1.37 \pm 0.06$ & $1.83 \pm 1.12$ & $1.19 \pm 0.61$ & $0.24 \pm 0.09$ \\
\hline & large intestine & $1.83 \pm 0.16$ & $0.78 \pm 0.13$ & $0.55 \pm 0.01$ & $0.96 \pm 0.25$ \\
\hline & muscle & $0.96 \pm 0.21$ & $0.57 \pm 0.06$ & $0.34 \pm 0.07$ & $0.13 \pm 0.04$ \\
\hline & bone & $1.52 \pm 0.19$ & $0.78 \pm 0.14$ & $0.36 \pm 0.02$ & $0.18 \pm 0.04$ \\
\hline & bladder & $3.89 \pm 0.40$ & $2.19 \pm 0.22$ & $7.48 \pm 0.68$ & $2.03 \pm 1.09$ \\
\hline & PC-3 PIP & $44.7 \pm 0.62$ & $57.1 \pm 19.55$ & $85.2 \pm 5.44$ & $96.6 \pm 18.7$ \\
\hline & PC-3 flu & $4.91 \pm 1.10$ & $2.25 \pm 1.62$ & $1.20 \pm 0.31$ & $0.42 \pm 0.07$ \\
\hline & PIP:flu & 9.1 & 25.4 & 71.1 & 231.1 \\
\hline & PIP:kidney & 0.5 & 0.5 & 0.7 & 1.3 \\
\hline & PIP:muscle & 46.6 & 100.2 & 250.5 & 743.0 \\
\hline & PIP:blood & 5.6 & 19.7 & 66.0 & 155.8 \\
\hline
\end{tabular}

${ }^{a}$ Biodistribution values are expressed as percent injected dose per gram \pm standard deviation. 